\title{
Relativistic nuclear model with point-couplings constrained by QCD and chiral symmetry ${ }^{\star}$
}

\author{
P. Finelli ${ }^{\mathrm{a}, \mathrm{d}}$, N. Kaiser ${ }^{\mathrm{b}}$, D. Vretenar $^{\mathrm{c}}, \mathrm{W}$. Weise ${ }^{\mathrm{d}, \mathrm{b}}$ \\ ${ }^{a}$ Physics Department, University of Bologna, and INFN - Bologna, I-40126 \\ Bologna, Italy \\ b Physik-Department, Technische Universität München, D-85747 Garching, \\ Germany \\ ${ }^{\mathrm{c}}$ Physics Department, Faculty of Science, University of Zagreb, 10000 Zagreb, \\ Croatia \\ ${ }^{\mathrm{d}} \mathrm{ECT}^{*}$, I-38050 Villazzano (Trento), Italy
}

\begin{abstract}
We derive a microscopic relativistic point-coupling model of nuclear many-body dynamics constrained by in-medium QCD sum rules and chiral symmetry. The effective Lagrangian is characterized by density dependent coupling strengths, determined by chiral one- and two-pion exchange and by QCD sum rule constraints for the large isoscalar nucleon self-energies that arise through changes of the quark condensate and the quark density at finite baryon density. This approach is tested in the analysis of the equations of state for symmetric and asymmetric nuclear matter, and of bulk and single-nucleon properties of finite nuclei. In comparison with purely phenomenological mean-field approaches, the built-in QCD constraints and the explicit treatment of pion exchange restrict the freedom in adjusting parameters and functional forms of density dependent couplings. It is shown that chiral (two-pion exchange) fluctuations play a prominent role for nuclear binding and saturation, whereas strong scalar and vector fields of about equal magnitude and opposite sign, induced by changes of the QCD vacuum in the presence of baryonic matter, generate the large effective spin-orbit potential in finite nuclei.
\end{abstract}

Key words: Relativistic mean field models, Chiral perturbation theory, QCD sum rules

PACS: 12.38.Bx, 21.65.+f, 21.60.-n, 21.30.Fe

\footnotetext{
* Work supported in part by BMBF, DFG and INFN
} 


\section{Introduction}

Concepts of effective field theory and density functional theory are at the basis of many successful nuclear structure models. Quantum Hadrodynamics (QHD), in particular, presents a field theoretical framework of Lorentzcovariant, meson-nucleon or point-coupling models of nuclear dynamics, that have been successfully employed in studies of a variety of nuclear phenomena [1]. Structure models based on the relativistic mean-field (RMF) approximation of QHD have been applied to describe spherical and deformed nuclei all over the periodic table [2]. More recently, this relativistic framework has also been used in studies of the structure of exotic nuclei with extreme isospin and close to the particle drip lines.

The effective Lagrangians of QHD consist of known long-range interactions constrained by symmetries and a set of generic short-range interactions. The most successful QHD models are phenomenological, with parameters adjusted to reproduce the nuclear matter equation of state and a set of global properties of spherical closed-shell nuclei. On the other hand, the description of nuclear many-body dynamics should ultimately be constrained by the underlying theory of the strong interactions - Quantum Chromodynamics (QCD). It is desirable to have a more explicit connection between low-energy QCD and nuclear phenomenology.

As pointed out in Refs. [1,3,4], the effective hadronic Lagrangians of QHD and related models are consistent with the symmetries of QCD: Lorentz invariance, parity invariance, electromagnetic gauge invariance, isospin and chiral symmetry. However, QHD calculations do not include pions explicitly. Effects of two-pion exchange are treated implicitly through a phenomenological scalar, isoscalar mean-field. Using the linear $\sigma$-model Lagrangian to construct a dynamical model for the $s$-wave $\pi \pi$ scattering amplitude, it has been shown that the exchange of a light scalar boson can be viewed as an approximate description of the exchange of correlated $s$-wave pion pairs [5]. While QCD symmetries constrain the effective QHD Lagrangians by restricting the form of possible interaction terms [4], the empirical data set of bulk and singleparticle properties of finite nuclei can only determine six or seven parameters in the general expansion of the effective Lagrangian in powers of the fields and their derivatives [6]. Such a general expansion is guided by the "naive dimensional analysis" (NDA) $[7,8,9,10]$. NDA tests the coefficients of the expansion for "naturalness", i.e. this method permits to control the orders of magnitude of the coupling constants. NDA can exclude some interaction terms because their couplings would be "unnatural", but it cannot determine the model parameters at the level of accuracy required for a quantitative analysis of nuclear structure data. 
The success of the relativistic nuclear mean-field phenomenology has been attributed to the large Lorentz scalar and four-vector nucleon self-energies, which are at the basis of all QHD models [3]. Even though the presence of large scalar and vector potentials cannot be directly verified experimentally, there is strong evidence, in particular from nuclear matter saturation and spinorbit splittings in finite nuclei, that the magnitude of each of these potentials is of the order of several hundred $\mathrm{MeV}$ in the nuclear interior. Studies based on finite-density QCD sum rules have shown how such large scalar and vector nucleon self-energies arise naturally from in-medium changes of the scalar quark condensate and the quark density $[11,12,13]$. In applications of QHD models to finite nuclei, however, the scalar and vector nucleon self-energies are treated in a purely phenomenological way. In some models they arise from the exchange of "sigma" and "omega" mesons, with adjustable strength parameters determined by the nuclear matter saturation and nuclear structure data. Additional nonlinear terms, or density-dependent coupling constants adjusted to Dirac-Brueckner self-energies in nuclear matter, are necessary in order to obtain a satisfactory nuclear matter equation of state and to reproduce the empirical bulk properties of finite nuclei.

The approach we adopt in the present work is based on the following conjectures that are believed to establish links between QCD, its symmetry breaking pattern, and the nuclear many-body problem:

- The nuclear ground state is characterized by large scalar and vector fields of approximately equal magnitude and opposite sign. These fields have their origin in the in-medium changes of the scalar quark condensate (the chiral condensate) and of the quark density.

- Nuclear binding and saturation arise prominently from chiral (pionic) fluctuations (reminiscent of van der Waals forces) in combination with Pauli blocking effects, superimposed on the condensate background fields and calculated according to the rules of in-medium chiral perturbation theory.

The first item has a direct connection with QCD sum rules at finite density. The second item is motivated by the observation that, at nuclear matter saturation density, the nucleon Fermi momentum $k_{f}$ and the pion mass $m_{\pi}$ represent comparable scales. We therefore include pions as explicit degrees of freedom in the description of nuclear many-body dynamics. Hammer and Furnsthal [14] pointed out earlier that an effective field theory of dilute Fermi systems must include pions for a proper description of the long-range physics. We support this statement by explicit calculations. While it is well known that the lowest order Fock terms from pion exchange are relatively small, the primary importance of chiral dynamics in nuclear systems is manifest in two-pion exchange processes combined with Pauli blocking effects which lead beyond 
standard mean-field approximations.

The broad spectral distribution of intermediate $\pi-\pi$ pairs with $J^{P}=0^{+}$, exchanged between nucleons, has large low-mass components starting at $2 m_{\pi}$. Hence their propagation in the presence of the nuclear Fermi sea is resolved at the Fermi momenta $k_{f} \simeq 2 m_{\pi}$ characteristic of nuclear matter. Guided by such considerations the nuclear matter problem has been investigated in Ref. [15] within the framework of chiral effective field theory. The calculations have been performed using in-medium chiral perturbation theory to three-loop order and incorporate the one-pion exchange Fock term, iterated one-pion exchange and irreducible two-pion exchange. The resulting nuclear matter equation of state is expressed as an expansion in powers of the Fermi momentum $k_{f}$. The expansion coefficients are functions of $k_{f} / m_{\pi}$, the dimensionless ratio of the two relevant small scales. The calculation involves one single momentum space cutoff $\Lambda$ which encodes NN-dynamics at short distances. This cutoff scale is the only free parameter. Its value can be adjusted to the energy per particle $\bar{E}\left(k_{f 0}\right)$ at saturation density. With $\Lambda \simeq 0.65 \mathrm{GeV}$ adjusted to $\bar{E}\left(k_{f 0}\right)=-15.3 \mathrm{MeV}$, the calculated equation of state gives the saturation density $\rho_{0}=0.178 \mathrm{fm}^{-3}$, the compression modulus $K_{0}=255 \mathrm{MeV}$, and the asymmetry energy $A\left(k_{f 0}\right)=$ $33.8 \mathrm{MeV}$ at saturation density. The corresponding momentum- and densitydependent single-particle potential $U\left(p, k_{f}\right)$ of nucleons in isospin-symmetric nuclear matter has been calculated in Ref. [16]. In particular, it has been shown that, at equilibrium nuclear matter density, chiral one- and two-pion exchanges generate an attractive nuclear mean-field for nucleons at the bottom of the Fermi sea, with a depth of $U\left(0, k_{f 0}\right)=-53.2 \mathrm{MeV}$, in good agreement with the depth of the empirical optical-model potential deduced by extrapolation from elastic nucleon-nucleus scattering data, and with the average nuclear potentials employed in shell-model and mean-field calculations for finite nuclei.

In Ref. [17] we have outlined an approach to the nuclear many-body problem, both for nuclear matter and finite nuclei, which emphasizes the connection with the condensate structure of the QCD ground state and pion dynamics based on spontaneous chiral symmetry breaking. A relativistic point coupling model has been used, with density dependent couplings governed by scales of low-energy QCD. We have shown that chiral fluctuations play a prominent role for nuclear binding and saturation, whereas strong scalar and vector fields of equal magnitude and opposite sign, induced by changes of the QCD vacuum in the presence of baryonic matter, drive the large spin-orbit splitting in finite nuclei. The magnitude of these condensate background nucleon self-energies has been determined by the leading order in-medium QCD sum rules.

In a different implementation, a model emphasizing the same connections to QCD symmetries and structure, was introduced and applied at the onebaryon-loop level to finite nuclei and nuclear matter in Ref. [18]. 
In this work we expand our previous studies and present a generalized and consistent version of the relativistic point-coupling model introduced in Ref. [17]. For instance, in the previous work following Ref. [15], it was still assumed that at nuclear matter saturation density the ratio of the scalar and vector condensate potentials, arising through changes in the quark condensate and the quark density at finite baryon density, equals -1 and that the nuclear binding and saturation result entirely from pionic (chiral) fluctuations. This approximation is relaxed in the present generalized approach and the effects of the condensate background fields at finite density are included already at the nuclear matter level. Eventually we will demonstrate that with a small number of parameters, determined by the underlying microscopic dynamics, and with minor fine-tuning of higher order corrections, it is possible to describe nuclear matter, as well as properties of finite nuclei, on a level comparable to phenomenological relativistic mean-field models.

In Sec. 2 we present the formalism and develop the relativistic point-coupling model constrained by QCD and chiral symmetry. An effective interaction, characterized by density dependent coupling parameters of the local fourpoint interaction terms, is derived in Sec. 3 and adjusted to the equation of state for symmetric and asymmetric nuclear matter. In Sec. 4 the model is employed in self-consistent calculations of ground-state properties of light and medium-heavy $N \approx Z$ nuclei. The calculated bulk properties and singlenucleon spectra are compared with experimental data and with results of the standard boson-exchange relativistic mean-field model. Summary and conclusions are presented in Sec. 5 .

\section{Point-coupling model}

\subsection{Lagrangian}

The relativistic point-coupling Lagrangian is built from basic densities and currents bilinear in the Dirac spinor field $\psi$ of the nucleon:

$$
\bar{\psi} \mathcal{O}_{\tau} \Gamma \psi \quad, \quad \mathcal{O}_{\tau} \in\left\{1, \tau_{i}\right\} \quad, \quad \Gamma \in\left\{1, \gamma_{\mu}, \gamma_{5}, \gamma_{5} \gamma_{\mu}, \sigma_{\mu \nu}\right\}
$$

Here $\tau_{i}$ are the isospin Pauli matrices and $\Gamma$ generically denotes the Dirac matrices. The interaction terms of the Lagrangian are products of these bilinears. In principle, a general effective Lagrangian can be written as a power series in the currents $\bar{\psi} \mathcal{O}_{\tau} \Gamma \psi$ and their derivatives. However, it is well known from numerous applications of relativistic mean-field models that properties of symmetric and asymmetric nuclear matter, as well as empirical ground state prop- 
erties of finite nuclei, constrain only the isoscalar-scalar, the isoscalar-vector, the isovector-vector, and to a certain extent the isovector-scalar channels.

The model that we consider in the present work includes the following fourfermion interaction vertices:

$$
\begin{array}{ll}
\text { isoscalar-scalar: } & (\bar{\psi} \psi)^{2} \\
\text { isoscalar-vector: } & \left(\bar{\psi} \gamma_{\mu} \psi\right)\left(\bar{\psi} \gamma^{\mu} \psi\right) \\
\text { isovector-scalar: } & (\bar{\psi} \vec{\tau} \psi) \cdot(\bar{\psi} \vec{\tau} \psi) \\
\text { isovector-vector: } & \left(\bar{\psi} \vec{\tau} \gamma_{\mu} \psi\right) \cdot\left(\bar{\psi} \vec{\tau} \gamma^{\mu} \psi\right) .
\end{array}
$$

Vectors in isospin space are denoted by arrows. The model is defined by the Lagrangian density

$$
\mathcal{L}=\mathcal{L}_{\text {free }}+\mathcal{L}_{4 \mathrm{f}}+\mathcal{L}_{\text {der }}+\mathcal{L}_{\text {em }}
$$

with the four terms specified as follows:

$$
\begin{aligned}
\mathcal{L}_{\text {free }}= & \bar{\psi}\left(i \gamma_{\mu} \partial^{\mu}-M\right) \psi, \\
\mathcal{L}_{4 \mathrm{f}}= & -\frac{1}{2} G_{S}(\hat{\rho})(\bar{\psi} \psi)(\bar{\psi} \psi) \\
& -\frac{1}{2} G_{V}(\hat{\rho})\left(\bar{\psi} \gamma_{\mu} \psi\right)\left(\bar{\psi} \gamma^{\mu} \psi\right) \\
& -\frac{1}{2} G_{T S}(\hat{\rho})(\bar{\psi} \vec{\tau} \psi) \cdot(\bar{\psi} \vec{\tau} \psi) \\
& -\frac{1}{2} G_{T V}(\hat{\rho})\left(\bar{\psi} \vec{\tau} \gamma_{\mu} \psi\right) \cdot\left(\bar{\psi} \vec{\tau} \gamma^{\mu} \psi\right), \\
\mathcal{L}_{\mathrm{der}}= & -\frac{1}{2} D_{S}(\hat{\rho})\left(\partial_{\nu} \bar{\psi} \psi\right)\left(\partial^{\nu} \bar{\psi} \psi\right) \\
& -\frac{1}{2} D_{V}(\hat{\rho})\left(\partial_{\nu} \bar{\psi} \gamma_{\mu} \psi\right)\left(\partial^{\nu} \bar{\psi} \gamma^{\mu} \psi\right)+\ldots, \\
\mathcal{L}_{\mathrm{em}}= & e A^{\mu} \bar{\psi} \frac{1+\tau_{3}}{2} \gamma_{\mu} \psi-\frac{1}{4} F_{\mu \nu} F^{\mu \nu} .
\end{aligned}
$$

This Lagrangian (2)-(6) is understood to be formally used in the mean-field approximation [19], with fluctuations encoded in density-dependent couplings $G_{i}(\hat{\rho})$ and $D_{i}(\hat{\rho})$, to be specified in detail later. In addition to the free nucleon Lagrangian $\mathcal{L}_{\text {free }}$ and the interaction terms contained in $\mathcal{L}_{4 \mathrm{f}}$, when applied to finite nuclei the model must include the coupling $\mathcal{L}_{\mathrm{em}}$ of the protons to the electromagnetic field $A^{\mu}$, and derivative terms contained in $\mathcal{L}_{\text {der }}{ }^{1}$. One can, of course, construct many more derivative terms of higher orders. In this way we

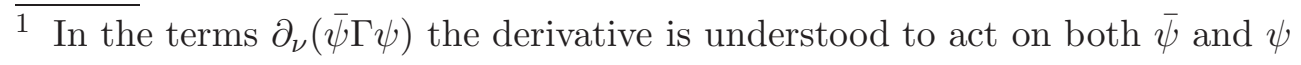


take into account leading effects of finite range interactions that are important for a quantitative fine-tuning of nuclear properties. In practice it will turn out that a single term $D_{S}\left(\partial_{\nu} \bar{\psi} \psi\right)\left(\partial^{\nu} \bar{\psi} \psi\right)$ is already sufficient to account for the detailed surface structure of the nuclei considered.

Our model Lagrangian is formally equivalent to the ones used in the standard relativistic mean-field point-coupling models of Refs. [20,21,8,9,10]. The underlying dynamics is, however, quite different. The parameters of the interaction terms in the standard point-coupling models have constant values adjusted to reproduce the nuclear matter equation of state and a set of global properties of spherical closed-shell nuclei. In order to describe properties of finite nuclei on a quantitative level, these models include also some higher order interaction terms, such as six-fermion vertices $(\bar{\psi} \psi)^{3}$, and eight-fermion vertices $(\bar{\psi} \psi)^{4}$ and $\left[\left(\bar{\psi} \gamma_{\mu} \psi\right)\left(\bar{\psi} \gamma^{\mu} \psi\right)\right]^{2}$. The model that we develop in this work includes only second order interaction terms. On the other hand, the coupling parameters are not constants, but rather functions of the nucleon density operator $\hat{\rho}$. Their functional dependence will be determined from finite-density QCD sum rules and in-medium chiral perturbation theory.

Medium dependent vertex functions have also been considered in the framework of relativistic mean-field meson-exchange models. In the density dependent relativistic hadron field (DDRH) models the medium dependence of the meson-nucleon vertices is expressed as a functional of the baryon field operators. The meson-nucleon vertex functions are determined either by mapping the nuclear matter Dirac-Brueckner nucleon self-energies in the local density approximation [19,22,23], or the parameters of an assumed phenomenological density dependence of the meson-nucleon couplings are adjusted to reproduce properties of symmetric and asymmetric nuclear matter and finite nuclei $[24,25]$. In practical applications of the DDRH models the meson-nucleon couplings are assumed to be functions of the baryon density $\psi^{\dagger} \psi$. In a relativistic framework the couplings can also depend on the scalar density $\bar{\psi} \psi$. Nevertheless, expanding in $\psi^{\dagger} \psi$ is the natural choice, for several reasons. The baryon density is connected to the conserved baryon number, unlike the scalar density for which no conservation law exists. The scalar density is a dynamical quantity, to be determined self-consistently by the equations of motion, and expandable in powers of the Fermi momentum. For the meson-exchange models it has been shown that the dependence on baryon density alone provides a more direct relation between the self-energies of the density-dependent hadron field theory and the Dirac-Brueckner microscopic self-energies [23]. Moreover, the pion-exchange contributions to the nucleon self-energy, as calculated using in-medium chiral perturbation theory, are directly given as expansions in powers of the Fermi momentum. Following these considerations, we express the coupling strengths of the interaction terms as functions of the baryon density, represented by the operator $\hat{\rho}=\psi^{\dagger} \psi$ in the rest frame of the many-body system. 


\subsection{Equation of motion and nucleon self-energies}

The single-nucleon Dirac equation is derived by variation of the Lagrangian (2) with respect to $\bar{\psi}$ :

$$
\left[\gamma_{\mu}\left(i \partial^{\mu}-V^{\mu}\right)-(M+S)\right] \psi=0
$$

where

$$
V^{\mu}=\Sigma^{\mu}+\vec{\tau} \cdot \vec{\Sigma}_{T}^{\mu}+\Sigma_{r}^{\mu}
$$

and

$$
S=\Sigma_{S}+\vec{\tau} \cdot \vec{\Sigma}_{T S}+\Sigma_{r S}
$$

with the nucleon self-energies defined by the following relations

$$
\begin{aligned}
\Sigma^{\mu}= & G_{V}\left(\bar{\psi} \gamma^{\mu} \psi\right)-e A^{\mu} \frac{1+\tau_{3}}{2} \\
\vec{\Sigma}_{T}^{\mu}= & G_{T V}\left(\bar{\psi} \vec{\tau} \gamma^{\mu} \psi\right), \\
\Sigma_{S}= & G_{S}(\bar{\psi} \psi)-D_{S} \square(\bar{\psi} \psi), \\
\vec{\Sigma}_{T S}= & G_{T S}(\bar{\psi} \vec{\tau} \psi), \\
\Sigma_{r S}= & -\frac{\partial D_{S}}{\partial \hat{\rho}}\left(\partial_{\nu} j^{\mu}\right) u_{\mu}\left(\partial^{\nu}(\bar{\psi} \psi)\right), \\
\Sigma_{r}^{\mu}= & \frac{u^{\mu}}{2}\left(\frac{\partial G_{S}}{\partial \hat{\rho}}(\bar{\psi} \psi)(\bar{\psi} \psi)+\frac{\partial G_{T S}}{\partial \hat{\rho}}(\bar{\psi} \vec{\tau} \psi) \cdot(\bar{\psi} \vec{\tau} \psi)\right. \\
& +\frac{\partial G_{V}}{\partial \hat{\rho}}\left(\bar{\psi} \gamma^{\mu} \psi\right)\left(\bar{\psi} \gamma_{\mu} \psi\right)+\frac{\partial G_{T V}}{\partial \hat{\rho}}\left(\bar{\psi} \vec{\tau} \gamma^{\mu} \psi\right) \cdot\left(\bar{\psi} \vec{\tau} \gamma_{\mu} \psi\right) \\
& \left.+\frac{\partial D_{S}}{\partial \hat{\rho}}\left(\partial^{\nu}(\bar{\psi} \psi)\right)\left(\partial_{\nu}(\bar{\psi} \psi)\right)\right) .
\end{aligned}
$$

We write $\hat{\rho} u^{\mu}=\bar{\psi} \gamma^{\mu} \psi$ and the four-velocity $u^{\mu}$ is defined as $\left(1-\boldsymbol{v}^{2}\right)^{-1 / 2}(1, \boldsymbol{v})$ where $\boldsymbol{v}$ is the three-velocity vector $(\boldsymbol{v}=0$ in the rest-frame of the nuclear system). In addition to the isoscalar-vector $\Sigma^{\mu}$, isoscalar-scalar $\Sigma_{S}$, isovectorvector $\vec{\Sigma}_{T}^{\mu}$ and isovector-scalar $\vec{\Sigma}_{T S}$ self-energies, the density dependence of the vertex functions produces the rearrangement contributions $\Sigma_{r S}$ and $\Sigma_{r}^{\mu}$. The 
rearrangement terms result from the variation of the vertex functionals with respect to the baryon fields in the density operator $\hat{\rho}$ (which coincides with the baryon density in the nuclear matter rest-frame). For a model with density dependent couplings, the inclusion of the rearrangement self-energies is essential for energy-momentum conservation and thermodynamical consistency (i.e. for the pressure equation derived from the thermodynamic definition and from the energy-momentum tensor) $[19,24]$.

The density dependence of the coupling strengths which determine the selfenergies (10-15), will be constrained by in-medium QCD sum rules and chiral pion dynamics, to be specified in detail in the following subsections 3.2 and 3.3.

When applied to nuclear matter or ground-state properties of finite nuclei, the point-coupling model is understood to be used in the mean-field approximation. The ground state of a nucleus with A nucleons is the antisymmetrized product of the lowest occupied single-nucleon self-consistent stationary solutions of the Dirac equation Eq. (7). The ground state energy is the sum of the single-nucleon energies plus a functional of proton and neutron scalar and particle densities.

\section{Nuclear matter equation of state}

\subsection{Framework}

In the translationally invariant infinite nuclear matter all terms involving the derivative couplings (5) drop out. The spatial components of the four-currents vanish, and the densities are calculated by taking expectation values

$$
\begin{aligned}
\rho_{s} & =\langle\Phi|\bar{\psi} \psi| \Phi\rangle=\rho_{s}^{p}+\rho_{s}^{n}, \\
\rho & =\left\langle\Phi\left|\bar{\psi} \gamma^{0} \psi\right| \Phi\right\rangle=\rho^{p}+\rho^{n}, \\
\rho_{s 3} & =\left\langle\Phi\left|\bar{\psi} \tau_{3} \psi\right| \Phi\right\rangle=\rho_{s}^{p}-\rho_{s}^{n}, \\
\rho_{3} & =\left\langle\Phi\left|\bar{\psi} \tau_{3} \gamma^{0} \psi\right| \Phi\right\rangle=\rho^{p}-\rho^{n},
\end{aligned}
$$

where $|\Phi\rangle$ is the nuclear matter ground state. The energy density $\epsilon$ and the pressure $P$ are derived from the energy-momentum tensor as

$$
\begin{aligned}
& \epsilon=\epsilon_{k i n}^{n}+\epsilon_{k i n}^{p}-\frac{1}{2} G_{S} \rho_{s}^{2}-\frac{1}{2} G_{T S} \rho_{s 3}^{2}+\frac{1}{2} G_{V} \rho^{2}+\frac{1}{2} G_{T V} \rho_{3}^{2} \\
& P=\tilde{E}^{p} \rho^{p}+\tilde{E}^{n} \rho^{n}-\epsilon_{k i n}^{p}-\epsilon_{k i n}^{n}+\frac{1}{2} G_{V} \rho^{2}+\frac{1}{2} G_{T V} \rho_{3}^{2}+\frac{1}{2} G_{S} \rho_{s}^{2}+\frac{1}{2} G_{T S} \rho_{s 3}^{2}
\end{aligned}
$$




$$
+\frac{1}{2} \frac{\partial G_{S}}{\partial \rho} \rho_{s}^{2} \rho+\frac{1}{2} \frac{\partial G_{V}}{\partial \rho} \rho^{3}+\frac{1}{2} \frac{\partial G_{T V}}{\partial \rho} \rho_{3}^{2} \rho+\frac{1}{2} \frac{\partial G_{T S}}{\partial \rho} \rho_{s 3}^{2} \rho .
$$

The particle density $\rho^{i}$ is related to the Fermi momentum $k_{f}^{i}$ in the usual way,

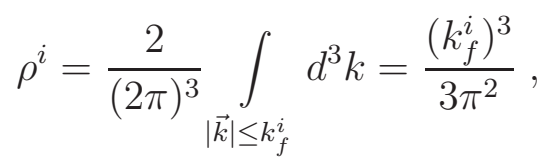

where the index $i=p, n$ refers to protons and neutrons, respectively. The

corresponding scalar densities are determined by the self-consistency relation

$$
\rho_{s}^{i}=\frac{2}{(2 \pi)^{3}} \int_{|\vec{k}| \leq k_{f}^{i}} d^{3} k \frac{M_{i}^{*}}{\sqrt{\vec{k}^{2}+\left(M_{i}^{*}\right)^{2}}}=\frac{M_{i}^{*}}{2 \pi^{2}}\left[k_{f}^{i} \tilde{E}^{i}-\left(M_{i}^{*}\right)^{2} \ln \frac{k_{f}^{i}+\tilde{E}^{i}}{M_{i}^{*}}\right],
$$

with

$$
\tilde{E}^{i}=\sqrt{\left(k_{f}^{i}\right)^{2}+\left(M_{i}^{*}\right)^{2}}
$$

and the effective nucleon masses

$$
M_{p, n}^{*}=M+G_{S} \rho_{s} \pm G_{T S} \rho_{s 3}
$$

The kinetic contributions to the energies of the protons and neutrons in nuclear matter are calculated from

$$
\epsilon_{k i n}^{i}=\frac{2}{(2 \pi)^{3}} \int_{|\vec{k}| \leq k_{f}^{i}} d^{3} k \sqrt{\vec{k}^{2}+\left(M_{i}^{*}\right)^{2}}=\frac{1}{4}\left[3 \tilde{E}^{i} \rho^{i}+M_{i}^{*} \rho_{s}^{i}\right], \quad i=p, n .
$$

Note that, in contrast to the energy density, rearrangement contributions appear explicitly in the expression for the pressure.

\subsection{QCD constraints}

We proceed now with a central theme of our work: establishing connections between the density-dependent point couplings in (10-15) and constraints from QCD. Two key features of low-energy, non perturbative QCD are at the origin of this discussion: the presence of a non-trivial vacuum characterized by strong condensates and the important role of pionic fluctuations governing 
the low-energy, long wavelength dynamics according to the rules imposed by spontaneously broken chiral symmetry.

The basic conjecture underlying our model is, consequently, that the nucleon isoscalar self-energies arise primarily through changes in the quark condensate and in the quark density at finite baryon density, together with chiral (pionic) fluctuations induced by one- and two-pion exchange interactions. In-medium QCD sum rules relate the changes of the scalar quark condensate and the quark density at finite baryon density, with the scalar and vector self-energies of a nucleon in the nuclear medium. In leading order which should be valid at densities below and around saturated nuclear matter, the condensate part of the scalar self-energy is expressed in terms of the density dependent chiral condensate as follows $[11,12,13]$ :

$$
\Sigma_{S}^{(0)}=-\frac{8 \pi^{2}}{\Lambda_{B}^{2}}\left[\langle\bar{q} q\rangle_{\rho}-\langle\bar{q} q\rangle_{0}\right]=-\frac{8 \pi^{2}}{\Lambda_{B}^{2}} \frac{\sigma_{N}}{m_{u}+m_{d}} \rho_{s} .
$$

The chiral vacuum condensate $\langle\bar{q} q\rangle_{0}$ is a measure of spontaneous chiral symmetry breaking in QCD. At a renormalization scale of about $1 \mathrm{GeV}$ (with quark masses $\left.m_{u}+m_{d} \simeq 12 \mathrm{MeV}\right)$ its value $[26]$ is $\langle\bar{q} q\rangle_{0} \simeq-(240 \mathrm{MeV})^{3} \simeq$ $-1.8 \mathrm{fm}^{-3}$. In order to appreciate the strength of this scalar condensate, note that its magnitude is more than a factor of ten larger than the baryon density in the bulk of a heavy nucleus. The difference between the vacuum condensate $\langle\bar{q} q\rangle_{0}$ and the one at finite density involves the nucleon sigma term, $\sigma_{N}=\left\langle N\left|m_{q} \bar{q} q\right| N\right\rangle$, to this order. Furthermore, $\Lambda_{B} \approx 1 \mathrm{GeV}$ is a characteristic scale (the Borel mass) roughly separating perturbative and non-perturbative domains in the QCD sum rule analysis. To the same order in the condensates with lowest dimension, the time component of the vector self-energy is

$$
\Sigma_{V}^{(0)}=\frac{64 \pi^{2}}{3 \Lambda_{B}^{2}}\left\langle q^{\dagger} q\right\rangle_{\rho}=\frac{32 \pi^{2}}{\Lambda_{B}^{2}} \rho
$$

where the quark baryon density is simply related to that of the nucleons by $\left\langle q^{\dagger} q\right\rangle_{\rho}=\frac{3}{2} \rho$.

When taken at the same leading order, the QCD sum rule analysis also identifies the free nucleon mass at $\rho=0$ according to Ioffe's formula [27], $M=-\frac{8 \pi^{2}}{\Lambda_{B}^{2}}\langle\bar{q} q\rangle_{0}$. Together with the Gell-Mann, Oakes, Renner relation $\left(m_{u}+m_{d}\right)\langle\bar{q} q\rangle_{0}=-m_{\pi}^{2} f_{\pi}^{2}$ (where $m_{\pi}$ is the pion mass and $f_{\pi}=92.4 \mathrm{MeV}$ is the pion decay constant), one finds

$$
\Sigma_{S}^{(0)}(\rho)=M^{*}(\rho)-M=-\frac{\sigma_{N} M}{m_{\pi}^{2} f_{\pi}^{2}} \rho_{s}
$$


and

$$
\Sigma_{V}^{(0)}(\rho)=\frac{4\left(m_{u}+m_{d}\right) M}{m_{\pi}^{2} f_{\pi}^{2}} \rho .
$$

Given these self-energies arising from the condensate background, the corresponding equivalent point-coupling strengths $G_{S, V}^{(0)}$ are simply determined by

$$
\Sigma_{S}^{(0)}=G_{S}^{(0)} \rho_{s}
$$

and

$$
\Sigma_{V}^{(0)}=G_{V}^{(0)} \rho
$$

The second important ingredient is the contribution to the nucleon self-energies from chiral fluctuations related to pion-exchange processes (primarily iterated one-pion exchange with small corrections from one-pion exchange Fock terms). While pion-exchange processes are not explicitly covered by standard Hartreetype mean field descriptions, their effects can nevertheless be re-expressed as density dependent corrections to the mean fields. The corresponding pointcoupling strengths, $G_{S, V}^{(\pi)}(\rho)$ etc., and their explicit density dependencies are calculated using in-medium chiral perturbation theory to a given loop order.

Combining effects from leading QCD condensates and pionic fluctuations, the strength parameters of the isoscalar four-fermion interaction terms in the Lagrangian (2) are:

$$
G_{S, V}(\rho)=G_{S, V}^{(0)}+G_{S, V}^{(\pi)}(\rho)
$$

For the isovector channels we assume in this work that only pionic (chiral) fluctuations contribute.

In Ref. [15] the nuclear matter equation of state (EOS) has been calculated using in-medium chiral perturbation theory up to three loop order in the energy density, expanded in powers of the Fermi momentum $k_{f}$ (modulo functions of $k_{f} / m_{\pi}$ ). The empirical saturation point, the nuclear matter incompressibility, and the asymmetry energy at saturation can be well reproduced at order $\mathcal{O}\left(k_{f}^{5}\right)$ in the chiral expansion with just one single momentum cut-off scale $\Lambda \simeq 0.65 \mathrm{GeV}$ which parameterizes short-distance physics. In this approach the nuclear matter saturation mechanism is entirely determined by in-medium pion-nucleon dynamics: the interplay between attraction from twopion exchange processes and the stabilizing effects of the Pauli principle. The implicit assumption made in the analysis of Ref. [15] is the following: there is 
no net contribution to the energy density around saturation from the nucleon isoscalar self-energies, $\Sigma_{S}^{(0)}$ and $\Sigma_{V}^{(0)}$, that arise through changes in the quark condensate and the quark density at finite baryon density. At first sight, this assumption appears to be unrealistic, given that in-medium QCD sum rules suggest individually large scalar and vector self-energies of about $300-400$ $\mathrm{MeV}$ in magnitude from the condensate background (see Eqs. (29)- (30)). On the other hand, the ratio

$$
\frac{\Sigma_{S}^{(0)}}{\Sigma_{V}^{(0)}}=-\frac{\sigma_{N}}{4\left(m_{u}+m_{d}\right)} \frac{\rho_{s}}{\rho}
$$

is indeed consistent with -1 , for typical values of the nucleon sigma term $\sigma_{N}$ and the current quark masses $m_{u, d}$, and around nuclear matter saturation density where $\rho_{s} \simeq \rho$ (as an example, take $\sigma_{N} \simeq 50 \mathrm{MeV}[28]$ and $m_{u}+m_{d} \simeq 12$ $\mathrm{MeV}[26]$ at a scale of $1 \mathrm{GeV})$. One should note of course that the QCD sum rule constraints implied by (29)-(30) and by the ratio (34) are not very accurate at a quantitative level. The leading-order Ioffe formula on which Eq. (29) relies has corrections from condensates of higher dimension which are not well under control. The estimated error in the ratio $\Sigma_{S}^{(0)} / \Sigma_{V}^{(0)} \simeq-1$ is about $20 \%$, given the uncertainties in the values of $\sigma_{N}$ and $m_{u}+m_{d}$. Nevertheless, the constraints implied by Eq. (34) give important hints for further orientation.

\subsection{Equation of state based on in-medium Chiral Perturbation Theory}

In this work the nuclear matter equation of state will be calculated in three steps. In the first approximation we follow the implicit assumption made in Ref. [15] that $\Sigma_{S}^{(0)}=-\Sigma_{V}^{(0)}$ in nuclear matter, and neglect the contribution of the condensate background self-energies to the nuclear matter equation of state (EOS). The density dependence of the strength parameters is determined by equating the isoscalar-scalar, the isoscalar-vector, the isovector-scalar, and

the isovector-vector self-energies Eqs. (10) - (15) in the single-nucleon Dirac equation (7) with those calculated using in-medium chiral perturbation theory:

$$
\begin{aligned}
G_{S}^{(\pi)} \rho_{s} & =\Sigma_{S}^{\mathrm{CHPT}}\left(k_{f}, \rho\right), \\
G_{V}^{(\pi)} \rho+\Sigma_{r}^{(\pi)} & =\Sigma_{V}^{\mathrm{CHPT}}\left(k_{f}, \rho\right), \\
G_{T S}^{(\pi)} \rho_{s 3} & =\Sigma_{T S}^{\mathrm{CHPT}}\left(k_{f}, \rho\right), \\
G_{T V}^{(\pi)} \rho_{3} & =\Sigma_{T V}^{\mathrm{CHPT}}\left(k_{f}, \rho\right),
\end{aligned}
$$

with

$$
\Sigma_{r}^{(\pi)}=\frac{1}{2} \frac{\partial G_{S}^{(\pi)}}{\partial \rho} \rho_{s}^{2}+\frac{1}{2} \frac{\partial G_{V}^{(\pi)}}{\partial \rho} \rho^{2}+\frac{1}{2} \frac{\partial G_{T S}^{(\pi)}}{\partial \rho} \rho_{s 3}^{2}+\frac{1}{2} \frac{\partial G_{T V}^{(\pi)}}{\partial \rho} \rho_{3}^{2},
$$


where the superscripts $\pi$ indicates that the density dependence of the couplings originates from pionic (chiral) fluctuations. The EOS of symmetric and asymmetric nuclear matter calculated in CHPT give, via the Hugenholtz-van Hove theorem, the sums $U_{(T)}\left(k_{f}, k_{f}\right)=\Sigma_{(T) S}^{\mathrm{CHPT}}\left(k_{f}, \rho\right)+\Sigma_{(T) V}^{\mathrm{CHPT}}\left(k_{f}, \rho\right)$ of the scalar and vector nucleon self-energies in the isoscalar and isovector channels at the Fermi surface $p=k_{f}$ up to two-loop order, generated by chiral oneand two-pion exchange [16]. The difference $\Sigma_{(T) S}^{\mathrm{CHPT}}\left(k_{f}, \rho\right)-\Sigma_{(T) V}^{\mathrm{CHPT}}\left(k_{f}, \rho\right)$ is calculated from the same pion-exchange diagrams via charge conjugation of the single particle potential in nuclear matter, as explained in Ref. [16]. Following a procedure similar to the determination of the nucleon-meson vertices of relativistic mean-field models from Dirac-Brueckner calculations [23], we neglect the momentum dependence of $\Sigma_{(T) S, V}^{C H P T}(p, \rho)$ and take their values at the Fermi surface $p=k_{f}$. A polynomial fit up to order $k_{f}^{5}$ is performed, and all four CHPT self-energies have the same functional form,

$$
\begin{aligned}
\Sigma\left(k_{f}, \lambda\right)= & {\left[c_{30}+c_{31} \lambda+c_{32} \lambda^{2}+c_{3 L} \ln \frac{m_{\pi}}{4 \pi f_{\pi} \lambda}\right] \frac{k_{f}^{3}}{M^{2}} } \\
& +c_{40} \frac{k_{f}^{4}}{M^{3}}+\left[c_{50}+c_{5 L} \ln \frac{m_{\pi}}{4 \pi f_{\pi} \lambda}\right] \frac{k_{f}^{5}}{M^{4}},
\end{aligned}
$$

where the dimensionless parameter $\lambda$ is related to the momentum cut-off scale by $\Lambda=2 \pi f_{\pi} \lambda$, and $f_{\pi}=92.4 \mathrm{MeV}$ denotes the pion decay constant. In Table 1 we display the coefficients of the expansion (40) of the CHPT self-energies for all four channels. The cut-off parameter $\Lambda=646 \mathrm{MeV}$ that has been adjusted to the nuclear matter saturation in Ref. [15], corresponds to $\lambda \simeq 1.113$. In order to determine the density dependence of the couplings from Eqs. (35) (38), the CHPT self-energies are re-expressed in terms of the baryon density $\rho=2 k_{f}^{3} / 3 \pi^{2}$ :

$$
\begin{aligned}
& \Sigma_{S}^{\mathrm{CHPT}}\left(k_{f}, \rho\right)=\left(c_{s 1}+c_{s 2} \rho^{\frac{1}{3}}+c_{s 3} \rho^{\frac{2}{3}}\right) \rho, \\
& \Sigma_{V}^{\mathrm{CHPT}}\left(k_{f}, \rho\right)=\left(c_{v 1}+c_{v 2} \rho^{\frac{1}{3}}+c_{v 3} \rho^{\frac{2}{3}}\right) \rho, \\
& \Sigma_{T S}^{\mathrm{CHPT}}\left(k_{f}, \rho\right)=\left(c_{t s 1}+c_{t s 2} \rho^{\frac{1}{3}}+c_{t s 3} \rho^{\frac{2}{3}}\right) \rho_{3}, \\
& \Sigma_{T V}^{\mathrm{CHPT}}\left(k_{f}, \rho\right)=\left(c_{t v 1}+c_{t v 2} \rho^{\frac{1}{3}}+c_{t v 3} \rho^{\frac{2}{3}}\right) \rho_{3} .
\end{aligned}
$$

The resulting expressions for the density dependent couplings of the pionic fluctuation terms are ${ }^{2}$

$$
G_{S}^{(\pi)}=c_{s 1}+c_{s 2} \rho^{\frac{1}{3}}+c_{s 3} \rho^{\frac{2}{3}}
$$

$\overline{2 \text { small }}$ differences between $\rho_{s}$ and $\rho$ at nuclear matter densities are neglected here 


$$
\begin{aligned}
& G_{V}^{(\pi)}=\bar{c}_{v 1}+\bar{c}_{v 2} \rho^{\frac{1}{3}}+\bar{c}_{v 3} \rho^{\frac{2}{3}} \\
& G_{T S}^{(\pi)}=c_{t s 1}+c_{t s 2} \rho^{\frac{1}{3}}+c_{t s 3} \rho^{\frac{2}{3}} \\
& G_{T V}^{(\pi)}=c_{t v 1}+c_{t v 2} \rho^{\frac{1}{3}}+c_{t v 3} \rho^{\frac{2}{3}} \\
& \bar{c}_{v 3}=\frac{1}{4}\left(3 c_{v 3}-c_{s 3}-\delta^{2}\left(c_{t s 3}+c_{t v 3}\right)\right)
\end{aligned}
$$

where $\delta=\left(\rho_{n}-\rho_{p}\right) /\left(\rho_{n}+\rho_{p}\right)$. For $\lambda \simeq 1.113$, the coefficients of the expansion of the CHPT self-energies in powers of the baryon density Eqs. (41) (44) are given in Table 2. In Fig. 1 the resulting equation of state of isospin symmetric nuclear matter, calculated from Eqs. (20) and (21), is compared with the CHPT nuclear matter EOS of Ref. [15]. In Table 3 we compare the corresponding nuclear matter properties at the saturation point: the binding energy per particle, the saturation density, the compression modulus, and the asymmetry energy. The point-coupling model, with the density dependence of the strength parameters of the second order interaction terms determined by pionic (chiral) fluctuations, nicely reproduces the nuclear matter EOS calculated using in-medium CHPT. Small differences arise mainly because in the mapping of the CHPT nucleon self-energies on the self-energies of the point coupling model in Eqs. (35) - (38), the momentum dependence of the former has been frozen to the values at the Fermi momentum. This is a well known problem that arises also in the determination of the meson-nucleon in-medium vertices from Dirac-Brueckner calculations of nucleon self-energies in nuclear matter [23]. For comparison in Fig. 1 we also include the microscopic manybody nuclear matter equation of state of Friedman and Pandharipande [29].

The importance of the rearrangement self-energies implemented through Eqs. (36) and (46) for a model with density dependent couplings is illustrated in Fig. 2, where we compare the EOS of isospin symmetric nuclear matter calculated with and without the rearrangement contributions in the Eq. (36) that determines the density dependence of the vertex of the isoscalar-vector interaction term. The difference is indeed very large. Without the rearrangement terms in Eq. (36) a saturation density of only $0.1 \mathrm{fm}^{-3}$ is obtained, and the binding energy per particle is $-6.85 \mathrm{MeV}$. Also the nuclear matter incompressibility is too low: $124 \mathrm{MeV}$. Obviously, only with inclusion of the rearrangement contributions in the isoscalar-vector self-energy it is possible to reproduce the nuclear matter EOS calculated in CHPT in an equivalent approach with density dependent point couplings.

At very low-densities, the CHPT equation of state (or any mean-field approach) cannot reproduce realistic calculations of $E / A$, for obvious reasons. The low density limit of the energy per particle is not given by a free Fermi gas of nucleons, but by a gas of clusters (deuterons, etc...). As a consequence, the low-density limit of $E / A$ approaches a constant as $k_{f} \rightarrow 0$. The effect of this 
shift is marginal, however, for the bulk of nuclear matter around saturation density.

\subsection{Constraints from QCD Sum Rules: leading orders}

The next step in our calculation of the nuclear matter EOS includes the contributions of the condensate background self-energies in the isoscalar-scalar and isoscalar-vector channels. In Ref. [17] we have shown that, even though in a first approximation the condensate potentials do not play a role in the saturation mechanism, they are indeed essential for the description of ground-state properties of finite nuclei.

The nuclear matter EOS is calculated from Eqs. (20) and (21). In this case, the strength parameters of the isoscalar-scalar and isoscalar-vector interaction terms have the form given in Eq. (33), i.e. they include contributions from condensate background fields. For the isovector channel, on the other hand, the assumption of the model is that only pionic (chiral) fluctuations contribute to the nucleon self-energies. In leading order of the in-medium QCD sum rules the nucleon isoscalar self-energies that arise through changes in the quark condensate and the quark density are linear functions of the corresponding scalar and baryon densities (see Eqs. (31) and (32)). To this order, therefore, the corresponding couplings $G_{S, V}^{(0)}$ should be density independent. Eq. (29) implies (identifying $M$ with the free nucleon mass):

$$
\Sigma_{S}^{(0)} \simeq-350 \mathrm{MeV} \frac{\sigma_{N}}{50 \mathrm{MeV}} \frac{\rho_{s}}{\rho_{0}}
$$

Evidently, the leading-order in-medium change of the chiral condensate is a source of a strong, attractive scalar field which acts on the nucleon in such a way as to reduce its mass in nuclear matter by more than $1 / 3$ of its vacuum value. Using (31) one estimates:

$$
G_{S}^{(0)} \simeq-11 \mathrm{fm}^{2} \frac{\sigma_{N}}{50 \mathrm{MeV}} \text { at } \rho_{s} \simeq \rho_{0}=0.16 \mathrm{fm}^{-3}
$$

A correspondingly smaller value of $G_{S}^{(0)}$ results if only a fraction of the nucleon mass $M$ is associated with the chiral condensate, leaving room for non-leading contributions from higher dimensional condensates. Since the QCD sum rules suggest that the ratio of the condensate scalar and vector self-energies is close to -1 , one expects roughly $G_{V}^{(0)} \simeq-G_{S}^{(0)}$.

In our model both $G_{S}^{(0)}$ and $G_{V}^{(0)}$ are parameters to be adjusted to "empirical" saturation properties of nuclear matter, as well as to ground-state properties 
of finite nuclei. Moreover, since the nuclear matter saturation mechanism is not any more exclusively determined by pionic (chiral) fluctuations, the momentum cut-off scale $\Lambda$ (or the dimensionless $\lambda$ ) is an additional parameter in our calculation of the nuclear matter EOS. At this preliminary stage, the three parameters: $G_{S}^{(0)}, G_{V}^{(0)}$ and $\Lambda$ are adjusted to reproduce "empirical" nuclear matter properties: $E / A=-16 \mathrm{MeV}(5 \%), \rho_{0}=0.153 \mathrm{fm}^{-3}(10 \%), K_{0}=250$ $\mathrm{MeV}(10 \%)$, and $a_{4}=33 \mathrm{MeV}(10 \%)$. The values in parentheses correspond to the error bars used in the fitting procedure.

The result of the corresponding least-squares fit is shown in Fig. 3 (EOS of isospin symmetric nuclear matter in comparison with the one calculated exclusively from chiral pion-exchange) and in the first row of Table 4 for $G_{S}^{(0)}=-7$ $\mathrm{fm}^{2}, G_{V}^{(0)}=7 \mathrm{fm}^{2}$, and $\Lambda=685 \mathrm{MeV}$. The EOS so obtained is not yet satisfac-

tory. The asymmetry energy at saturation is too high, and the values of $G_{S}^{(0)}$ and $G_{V}^{(0)}$ are smaller than the leading order QCD sum rule estimate Eq. (50). This results in the relatively large Dirac effective mass $M^{*} / M=0.75$. A high Dirac mass in turn indicates that the effective single-nucleon spin-orbit potential is too weak to reproduce the empirical energy spacings between spin-orbit partner states in finite nuclei. Stronger background scalar and vector fields are required in order to drive the large spin-orbit splittings in finite nuclei.

Notice however that, even though $G_{S}^{(0)}$ and $G_{V}^{(0)}$ have been varied independently, the minimization procedure prefers to balance the contributions from the corresponding large scalar and vector self-energies so that their sum tends to vanish. There is already enough binding from pionic fluctuations alone, and therefore $\Sigma_{S}^{(0)}=-\Sigma_{V}^{(0)}$ represents a very good approximation for the condensate potentials. This remarkable feature prevails as we now proceed with fine-tuning improvements.

\subsection{Corrections of higher order}

Up to this point the nucleon self-energies are evaluated at two-loop level including terms of order $k_{f}^{5}$. At order $k_{f}^{6}\left(\propto \rho^{2}\right)$, several additional effects appear. First, condensates of higher dimension enter, such as $\langle\bar{q} \Gamma q \bar{q} \Gamma q\rangle,\left\langle q^{\dagger} q\right\rangle^{2}$ and the gluon condensate, the detailed in-medium dependence of which is difficult to estimate. Secondly, four-loop CHPT contributions to the energy density (three-loop in the self energies) introduce genuine 3-body interactions. All those effects combine to produce the $\mathcal{O}\left(k_{f}^{6}\right)$ correction in the energy per particle. Our chiral counting conjecture is that these terms should be subleading in the $k_{f}$-expansion. In estimating those contributions, we will take a pragmatic point of view. Given the density-independent leading condensate terms $G^{(0)}$ 
and the CHPT couplings $G^{(\pi)}(\rho)$ evaluated to $\mathcal{O}\left(k_{f}^{5}\right) \propto \rho^{\frac{2}{3}}$, we generalize

$$
G(\rho)=G^{(0)}+G^{(\pi)}(\rho)+\delta G^{(1)}(\rho)
$$

adding terms $\delta G^{(1)}=g^{(1)} \rho$ and let the constant $g^{(1)}$ be determined by a least squares fit to properties of nuclear matter and finite nuclei. At first sight, the corrections $\delta G_{S}^{(1)}, \delta G_{V}^{(1)}$, etc... would then introduce several additional parameters, undermining our startegy to keep the number of freely adjustable fine-tuning constants as small as possible. For the scalar self-energy a primary uncertainty arises from the four-quark condensate and its assumed factorization into the form $\langle\bar{q} q\rangle^{2}$, as discussed in detail in Ref. [13]. While the in-medium values of condensates such as $\langle\bar{q} \Gamma q \bar{q} \Gamma q\rangle$ are not well determined, it nevertheless appears that only a weak or moderate density dependence is consistent with known nuclear phenomenology. We do not have to be much concerned about this issue since our approach explicitly includes scalar $\pi-\pi$ fluctuations (up to order $k_{f}^{5}$ in the energy density), which should account for a large part of the four quark condensate effects. Remaining short-distance effects can be absorbed in $\delta G^{(1)}$.

As a first option, assume that the only non-vanishing $\delta G^{(1)}$ is $\delta G_{V}^{(1)}=g_{V}^{(1)} \rho$, and determine the constant $g_{V}^{(1)}$. For $G_{S}^{(0)}=-12 \mathrm{fm}^{2}, G_{V}^{(0)}=11 \mathrm{fm}^{2}, g_{V}^{(1)}=$ $-3.9 \mathrm{fm}^{5}$ and $\Lambda=600 \mathrm{MeV}$, determined in a least-squares fit to the "empirical" nuclear matter input, the EOS resulting is displayed in Fig. 3 and the nuclear matter properties at saturation are listed in the second row of Table 4. This nuclear matter EOS is actually quite satisfactory, with realistic values of the binding energy, saturation density and a low effective Dirac mass. The only exception is a relatively large nuclear matter incompressibility. Calculations of the excitation energies of isoscalar giant monopole resonances in spherical nuclei in the time-dependent relativistic mean-field framework [30], and in the relativistic random-phase approximation [31], suggest that the nuclear matter incompressibility modulus should be in the range $K_{0} \approx 250-270 \mathrm{MeV}$. A value of $K_{0}$ in this range, however, can only be obtained by including the next higher order non-linear self-interaction term in the expansion of $\Sigma_{V}$ or $\Sigma_{S}$, i.e. a term proportional to $\rho^{3}$. This feature is also well known in standard meson-exchange and point-coupling relativistic mean-field models. In the present analysis we do not aim for a detailed description of nuclear properties that depend crucially on the incompressibility, and we also wish to keep the number of adjustable parameters at minimum, so the density dependence of $G_{V}$ is parameterized as

$$
G_{V}(\rho)=G_{V}^{(0)}+G_{V}^{(\pi)}(\rho)+g_{V}^{(1)} \rho
$$

The resulting couplings of the condensate background fields $\left(G_{S}^{(0)}=-12 \mathrm{fm}^{2}\right.$ and $G_{V}^{(0)}=11 \mathrm{fm}^{2}$ ) are remarkably close to the prediction (50) of the leading 
order in-medium QCD sum rules. The scalar and the vector "condensate" selfenergies $\Sigma_{S}^{(0)}=G_{S}^{(0)} \rho_{s}$ and $\Sigma_{V}^{(0)}=G_{V}^{(0)} \rho$ follow the expectation $\Sigma_{S}^{(0)} \simeq-\Sigma_{V}^{(0)}$ to within $5 \%$ at saturation density, even without this condition being preimposed. The large isoscalar condensate background self-energies in turn lead to a relatively low effective Dirac mass, crucial for the empirical spin-orbit splittings in finite nuclei. Finally, the cut-off $\Lambda=600 \mathrm{MeV}$ differs by less than $10 \%$ from the value $(646 \mathrm{MeV})$ obtained when the nuclear matter EOS results exclusively from one- and two-pion exchange between nucleons [15].

The $\delta G_{V}^{(1)}=g_{V}^{(1)} \rho$ term acts like a three-body force in the energy density. Its effect is relatively small: at saturation density, $\delta G_{V}^{(1)} / G_{V}^{(0)} \simeq 0.05$. Splitting $\delta G^{(1)}$ between scalar and vector parts (e.g. by choosing $\delta G_{V}^{(1)} \simeq \delta G_{S}^{(1)} \simeq$ $-2 \mathrm{fm}^{-5}$ ) would induce only marginal changes.

\subsection{Asymmetric nuclear matter}

The energy per particle of asymmetric nuclear matter can be expanded about the equilibrium density $\rho_{\text {sat }}$ in a Taylor series in $\rho$ and $\delta$ [32]

$$
E(\rho, \delta)=E(\rho, 0)+S_{2}(\rho) \delta^{2}+S_{4}(\rho) \delta^{4}+\cdots
$$

where $\delta=\left(\rho_{n}-\rho_{p}\right) /\left(\rho_{n}+\rho_{p}\right)$, and

$$
\begin{aligned}
E(\rho, 0) & =-a_{v}+\frac{K_{0}}{18 \rho_{\text {sat }}^{2}}\left(\rho-\rho_{\text {sat }}\right)^{2}+\ldots, \\
S_{2}(\rho) & =a_{4}+\frac{p_{0}}{\rho_{\text {sat }}^{2}}\left(\rho-\rho_{\text {sat }}\right)+\frac{\Delta K_{0}}{18 \rho_{\text {sat }}^{2}}\left(\rho-\rho_{\text {sat }}\right)^{2}+\cdots .
\end{aligned}
$$

The empirical value at saturation density is $S_{2}\left(\rho_{\text {sat }}\right)=a_{4}=30 \pm 4 \mathrm{MeV}$. The parameter $p_{0}$ defines the linear density dependence of the symmetry energy, and $\Delta K_{0}$ is the correction to the incompressibility. The contribution of the term $S_{4}(\rho) \alpha^{4}$ in (53) is very small in ordinary nuclei and it will be neglected in the present work. In a recent analysis of neutron radii in non-relativistic and covariant mean-field models [33], Furnstahl has studied the linear correlation between the neutron skin and the asymmetry energy. In particular, he has shown that there is a very strong linear correlation between the neutron skin thickness in ${ }^{208} \mathrm{~Pb}$ and the individual parameters that determine the asymmetry energy $S_{2}(\rho): a_{4}, p_{0}$ and $\Delta K_{0}$. The empirical value of the difference between neutron and proton $r m s$ radii $r_{n}-r_{p}$ in ${ }^{208} \mathrm{~Pb}(0.20 \pm 0.04 \mathrm{fm}$ from proton scattering data [34], and $0.19 \pm 0.09 \mathrm{fm}$ from the alpha scattering excitation

of the isovector giant dipole resonance [35]) places the following constraints 
on the values of the parameters of the symmetry energy: $a_{4} \approx 30-34 \mathrm{MeV}$, $2 \mathrm{MeV} / \mathrm{fm}^{3} \leq p_{0} \leq 4 \mathrm{MeV} / \mathrm{fm}^{3}$, and $-200 \mathrm{MeV} \leq \Delta K_{0} \leq-50 \mathrm{MeV}$.

In Fig. 4 we plot the asymmetry energy $S_{2}(\rho)$ as function of the baryon density for the three steps of our calculation of the nuclear matter EOS: first, using only chiral one- and two-pion exchange calculated in CHPT, next with the inclusion of leading order isoscalar condensate background nucleon self-energies that arise through changes in the quark condensate and the quark density at finite baryon density, and finally with the non-linear contribution $\delta G_{V}^{(1)}$ to the vector condensate nucleon self-energy. The three curves can be compared, for instance, with the asymmetry energy obtained in the non-relativistic Brueckner-Hartree-Fock calculation of Ref. [36], or with the asymmetry energy of the relativistic meson-exchange model with a phenomenological density dependence of the meson-nucleon couplings in Ref. [25].

In Fig. 5 we display the energy per particle of neutron matter as a function of the neutron density. The results obtained from chiral one- and two-pion exchange between nucleons, by adding the isoscalar condensate background nucleon self-energies linear in the corresponding densities, and finally by including the non-linear "3-body" contribution to the isoscalar vector self-energy, are shown in comparison with the microscopic many-body neutron matter equation of state of Friedman and Pandharipande [29].

These comparisons show a qualitatively correct trend in both the asymmetry energy and the neutron matter equation of state, but refinements of the density dependent isovector couplings are obviously still necessary.

Of course, one cannot expect to reproduce the behaviour at very low neutron density in a perturbative approach. The very large neutron-neutron scattering length requires a partial resummation which effectively renormalizes the slope of the $k_{f}^{2}$-term in $E_{N} / N$ by roughly a factor of one-half $[33,37]$.

\subsection{Intermediate summary and discussions}

While the quantitative details for the terms contributing to the scalar and vector self-energies can be reconstructed from the previous sections using Tables 1 and 2, it is instructive to observe systematic trends in these self-energies when expanded in powers of $k_{f}$ (or equivalently, in powers of $\rho^{\frac{1}{3}}$ ). We focus on symmetric nuclear matter and write

$$
\Sigma_{S, V}=\Sigma_{S, V}^{(0)}+\Sigma_{S, V}^{(\pi)}+\delta \Sigma_{S, V}
$$


combining the leading condensate terms $\Sigma_{S, V}^{(0)}$, the chiral (pionic) terms $\Sigma_{S, V}^{(\pi)}$ calculated to order $k_{f}^{5}$, and possible corrections of higher order summarized in $\delta \Sigma_{S, V}$. We introduce the saturation density $\rho_{0}=0.16 \mathrm{fm}^{-3}$ as a convenient scale and write the condensate terms as

$$
\begin{aligned}
& \Sigma_{S}^{(0)}(\rho) \simeq-0.35 \mathrm{GeV} \frac{\left|G_{S}^{(0)}\right|}{11 \mathrm{fm}^{2}}\left(\frac{\rho_{s}}{\rho_{0}}\right), \\
& \Sigma_{V}^{(0)}(\rho) \simeq+0.35 \mathrm{GeV} \frac{\left|G_{V}^{(0)}\right|}{11 \mathrm{fm}^{2}}\left(\frac{\rho}{\rho_{0}}\right) .
\end{aligned}
$$

We note that with $\left|G_{S}^{(0)}\right|$ chosen less than $5 \%$ larger than $G_{V}^{(0)}$, this just compensates for the difference between the baryon density $\rho$ and the scalar density $\rho_{s}<\rho$ such that $\Sigma_{S}^{(0)} \simeq-\Sigma_{V}^{(0)}$ results at $\rho=\rho_{0}$. The "best-fit" values $G_{S}^{(0)} \simeq-12 \mathrm{fm}^{2}$ and $G_{V}^{(0)} \simeq+11 \mathrm{fm}^{2}$ are thus perfectly consistent with QCD sum rule expectations: a non-trivial result. The pionic fluctuations from oneand two-pion exchange processes have the following approximate pattern:

$$
\Sigma_{S, V}^{(\pi)}(\rho) \simeq-75 \mathrm{MeV}\left(\frac{\rho}{\rho_{0}}\right)\left[1+d_{1}\left(\frac{\rho}{\rho_{0}}\right)^{\frac{1}{3}}+d_{2}\left(\frac{\rho}{\rho_{0}}\right)^{\frac{2}{3}}\right]
$$

where $d_{1}$ varies between -0.61 (scalar) and -0.65 (vector), and $d_{2} \simeq-0.17$ for both cases. Finally, the higher order corrections are summarized as

$$
\delta \Sigma_{S}+\delta \Sigma_{V} \simeq-20 \mathrm{MeV}\left(\frac{\rho}{\rho_{0}}\right)^{2}
$$

where the detailed decomposition into scalar and vector part turns out not to be relevant.

The leading attraction at $\mathcal{O}\left(k_{f}^{3}\right)$ in $\Sigma_{S, V}^{(\pi)}$ depends on the cut-off scale $\Lambda \equiv$ $2 \pi f_{\pi} \lambda$ which distinguishes "active" two-pion exchange dynamics at long and intermediate distances from short-distance (high momentum) dynamics. Details at short distance scales (related to the intrinsic structure of the nucleons and their short-range interactions) are not resolved as long as the Fermi momentum $k_{f}$ is much smaller than $4 \pi f_{\pi}$. The unresolved short-distance information can then be translated into an equivalent (density-independent) four-point vertex which generates self-energy terms linear in the density at the mean-field level. 


\subsection{Comparison with the Dirac-Brueckner G matrix}

At this point it is instructive to compare the density-dependence of our selfenergies $\Sigma_{S, V}(\rho)$ with that of the scalar and the vector self-energies resulting from full Dirac-Brueckner calculations based on realistic NN-interactions. We refer here explicitly to a calculation, reported in Ref. [38], which uses the Bonn A potential. With this potential, the nuclear matter saturation density comes out somewhat higher $\left(\rho_{\text {sat }} \simeq 0.185 \mathrm{fm}^{-3}\right)$ than the one of our best fit. Simulating this higher saturation density in our approach requires weakening the condensate mean fields $\Sigma_{S, V}^{(0)}$ by about $25 \%$, but with no changes in the pionic terms $\Sigma_{S, V}^{(\pi)}$. After this readjustment, the difference in the $k_{f}$-dependences between our $\Sigma_{S}$ and $\Sigma_{V}$ and those resulting from the Dirac-Bruckner calculation is less than $10 \%$ over the entire range of densities from $0.5 \rho_{0}$ to $2.5 \rho_{0}$ (see Fig. 6).

This is a remarkable observation: it appears that in-medium chiral perturbation theory at two-loop order, with a cut-off scale $\Lambda \simeq 0.6 \mathrm{GeV}$ that converts unresolved short-distance dynamics at momenta beyond $\Lambda$ effectively into contact terms, generates quantitatively similar in-medium nucleon self energies as a full Dirac-Brueckner calculation, when requiring that both approaches reproduce the same nuclear matter saturation point. The reasoning behind this observation can presumably be traced back to the separation of scales at work, a key element of chiral effective field theory on which our approach is based. One- and two-pion exchange in-medium dynamics at momentum scales comparable to $k_{f}$ are treated explicitly. Our in-medium CHPT approach includes all terms (ladders and others) to three-loop order in the energy density, a prominent one being iterated one-pion exchange. The non-trivial $k_{f}$ dependence in the self-energies (beyond "trivial" order $k_{f}^{3}$ ) reflects the action of the Pauli principle in these processes. Such features are also present in leading orders of the Brueckner ladder summation which includes, for example, Pauli blocking effects on iterated one-pion exchange. These terms produce the characteristic $k_{f}^{4}$-dependence of the energy per particle. On the other hand, the iteration of short-distance interactions to all orders in the Brueckner ladder, involves intermediate momenta much larger than $k_{f}$ and generates similar

self-energy pieces (proportional to density $\rho$ ) as a contact interaction reflecting the high-momentum scale $\Lambda$. Note that this contact interaction must not be iterated as it already represents the full short-distance T-matrix information.

The reasoning here has a close correspondence to related studies in Ref. [39]. 


\section{Finite nuclei}

In this section the relativistic nuclear point-coupling model, constrained by in-medium QCD sum rules and chiral perturbation theory, is applied in calculations of ground state properties of finite nuclei. In the mean-field approximation the ground state of a nucleus with A nucleons is represented by the antisymmetrized product of the lowest occupied single-nucleon stationary solutions of the Dirac equation (7). The calculation is self-consistent in the sense that the nucleon self-energies are functions of nucleon densities and currents, calculated from the solutions of Eq. (7). The mean-field approach to nuclear structure represents an approximate implementation of Kohn-Sham density functional theory (DFT) [40,41,42], which is successfully employed in the treatment of the quantum many-body problem in atomic, molecular and condensed matter physics. At the basis of the DFT approach are energy functionals of the ground-state density. In relativistic mean-field models, these become functionals of the ground-state scalar density and of the baryon current. The scalar and vector self-energies play the role of local relativistic Kohn-Sham potentials [1,3]. The mean-field models approximate the exact energy functional, which includes all higher-order correlations, with powers and gradients of densities, with the truncation determined by power counting [3].

In this work we only consider spherical even-even nuclei. Because of timereversal invariance the spatial components of the four-currents vanish, and the nucleon self-energies (reduced to the time component $\Sigma^{0}$ of the vector terms $\Sigma^{\mu}$ ) read:

$$
\begin{aligned}
\Sigma^{0} & =G_{V} \rho-V_{C}, \\
\Sigma_{T}^{0} & =G_{T V} \rho_{3}, \\
\Sigma_{S} & =G_{S} \rho_{s}+D_{S} \triangle \rho_{s}, \\
\Sigma_{T S} & =G_{T S} \rho_{s 3} \\
\Sigma_{r S} & =\frac{\partial D_{S}}{\partial \rho}\left(\nabla \rho_{s}\right) \cdot(\nabla \rho), \\
\Sigma_{r}^{0} & =\frac{1}{2}\left\{\frac{\partial G_{S}}{\partial \rho} \rho_{s}^{2}+\frac{\partial G_{T S}}{\partial \rho} \rho_{s 3}^{2}+\frac{\partial G_{V}}{\partial \rho} \rho^{2}+\frac{\partial G_{T V}}{\partial \rho} \rho_{3}^{2}-\frac{\partial D_{S}}{\partial \rho}\left(\nabla \rho_{s}\right) \cdot\left(\nabla \rho_{s}\right)\right\},
\end{aligned}
$$

where $V_{C}$ is the Coulomb potential. In the mean-field approximation the isoscalar-scalar, isoscalar-vector, isovector-scalar and isovector-vector ground state densities are calculated from the Dirac wave functions $\psi_{k}$ of the occupied positive-energy orbits as: 


$$
\begin{gathered}
\rho_{s}=\sum_{k=1}^{A} \bar{\psi}_{k} \psi_{k}, \\
\rho=\sum_{k=1}^{A} \psi_{k}^{\dagger} \psi_{k}, \\
\rho_{s 3}=\sum_{k=1}^{A} \bar{\psi}_{k} \tau_{3} \psi_{k}, \\
\rho_{3}=\sum_{k=1}^{A} \psi_{k}^{\dagger} \tau_{3} \psi_{k},
\end{gathered}
$$

respectively. The expression for the ground state energy of a nucleus with A nucleons reads:

$$
\begin{aligned}
E= & \sum_{k=1}^{A} \epsilon_{k}-\frac{1}{2} \int d^{3} x\left[G_{S} \rho_{s}^{2}+G_{T S} \rho_{s 3}^{2}+G_{V} \rho^{2}++G_{T V} \rho_{3}^{2}+\rho^{p} V_{C}\right. \\
& +\frac{\partial G_{S}}{\partial \rho} \rho_{s}^{2} \rho+\frac{\partial G_{V}}{\partial \rho} \rho^{3}+\frac{\partial G_{T V}}{\partial \rho} \rho_{3}^{2} \rho+\frac{\partial G_{T S}}{\partial \rho} \rho_{s 3}^{2} \rho+D_{S} \rho_{s} \triangle \rho_{s} \\
& \left.-\frac{\partial D_{S}}{\partial \rho}\left(\nabla \rho_{s}\right) \cdot\left(\nabla \rho_{s}\right) \rho+2 \frac{\partial D_{S}}{\partial \rho}\left(\nabla \rho_{s}\right) \cdot(\nabla \rho) \rho_{s}\right]
\end{aligned}
$$

where $\epsilon_{k}$ denotes the single-nucleon energies. In addition, for open shell nuclei pairing correlations are included in the simple BCS approximation [43]. After obtaining the solution of the self-consistent Dirac equation, the microscopic estimate for the center-of-mass correction

$$
E_{c m}=-\frac{\left\langle\vec{P}_{c m}^{2}\right\rangle}{2 A M}
$$

is subtracted from the total binding energy. Here $\vec{P}_{c m}^{2}$ is the squared total momentum of the nucleus with $A$ nucleons.

The details of the calculated properties of finite nuclei will, of course, depend on the fine-tuning of the coupling parameters. The density-dependent couplings $G_{S}, G_{V}, G_{T S}$ and $G_{T V}$ are constrained by in-medium QCD sum rules and chiral perturbation calculations of one- and two-pion exchange diagrams, as described in the previous section. For finite nuclei we must also determine the coupling parameter of the derivative term: $D_{S}$. Dimensional considerations suggest the following ansatz

$$
D_{S}(\rho)=\frac{G_{S}(\rho)}{\mathcal{M}^{2}}
$$

where $\mathcal{M}$ is a characteristic mass scale for a given spin-isospin channel. There is no deeper reason, however, for the parameters of the derivative terms to have 
the same density dependence as the coupling parameters of the four-fermion interaction terms. The simplest option, followed in this work, is to introduce only $D_{S}$ and treat it as a density-independent adjustable parameter. In this case the remaining rearrangement contribution to the vector-self energy becomes, of course, much simplified. As it has been emphasized by Serot and Furnstahl [6], the empirical data set of bulk and single-particle properties of finite nuclei can only constrain six or seven parameters in the general expansion of the effective Lagrangian in powers of the fields and their derivatives. In particular, only one parameter of the derivative terms can be determined by the binding energies and radii of spherical nuclei. We adjust the single remaining surface parameter $D_{S}$ of the isoscalar-scalar derivative term to properties of light and medium-heavy $N \approx Z$ nuclei. This approximation, which was first used by Serot and Walecka in Ref. [44], implies that the isoscalar-vector, the isovector-scalar and the isovector-vector interactions are considered to be purely contact interactions (no gradient terms).

Before we present the results of full model calculations for finite nuclei, it is instructive to consider separately the contributions of chiral pion dynamics and condensate background self-energies to properties of finite nuclei. In the first step we have calculated the ground states of ${ }^{16} \mathrm{O}$ and ${ }^{40} \mathrm{Ca}$ by using the coupling parameters determined by the nuclear matter EOS of Ref. [15] (see Fig. 1): $G_{S}(\rho)=G_{S}^{(\pi)}(\rho), G_{V}(\rho)=G_{V}^{(\pi)}(\rho), G_{T S}(\rho)=G_{T S}^{(\pi)}(\rho), G_{T V}(\rho)=G_{T V}^{(\pi)}(\rho)$, $\Lambda=646.3 \mathrm{MeV}$, while the couplings to the condensate background fields are set to zero. In this case the nuclear dynamics is completely determined by chiral (pionic) fluctuations. The calculated total binding energies are already within $5-8 \%$ of the experimental values, but the radii of the two nuclei are too small (by about $0.2 \mathrm{fm}$ ). This is because the spin-orbit partners $\left(1 p_{3 / 2}, 1 p_{1 / 2}\right)$ and $\left(1 d_{5 / 2}, 1 d_{3 / 2}\right)$ are practically degenerate. In Fig. 7 we display the calculated neutron and proton single-particle levels of ${ }^{16} \mathrm{O}$ and ${ }^{40} \mathrm{Ca}$. The energies of the degenerate doublets are close to the empirical positions of the centroids of the spin-orbit partner levels, and even the calculated energies of the $s$-states are realistic. This is a very interesting result. The chiral pion dynamics provides the saturation mechanism and binding of nuclear matter, but not the strong spin-orbit force. The inclusion of the isoscalar-scalar derivative term has some effect on the calculated radii but it cannot remove the degeneracy of the spinorbit doublets.

The spin-orbit potential plays a central role in nuclear structure. It is at the basis of the nuclear shell model, and its inclusion is essential in order to reproduce the experimentally established magic numbers. In non-relativistic models based on the mean-field approximation, the spin-orbit potential is included in a purely phenomenological way, introducing the strength of the spin-orbit interaction as an additional parameter. Its value is usually adjusted to the experimental spin-orbit splittings in spherical nuclei, for example ${ }^{16} \mathrm{O}$. On the other hand, in the relativistic description of the nuclear many-body prob- 
lem, the spin-orbit interaction arises naturally from the scalar-vector Lorentz structure of the effective Lagrangian, and relativistic models reproduce the empirical spin-orbit splittings. In the first order approximation, and assuming spherical symmetry, the spin-orbit term of the effective single-nucleon potential can be written as

$$
V_{\text {s.o. }}=\frac{1}{2 M^{2}}\left(\frac{1}{r} \frac{\partial}{\partial r} V_{l s}(r)\right) \boldsymbol{l} \cdot \boldsymbol{s},
$$

where the large spin-orbit potential $V_{l s}$ arises from the difference of the vector and scalar potentials, $V^{0}-S \sim 0.7 \mathrm{GeV}[2,45]$. Explicitly,

$$
V_{l s}=\frac{M}{M_{e f f}}\left(V^{0}-S\right) .
$$

where $M_{\text {eff }}$ is an effective mass specified as [2]

$$
M_{e f f}=M-\frac{1}{2}\left(V^{0}-S\right)
$$

The isoscalar nucleon self-energies generated by pion exchange are not sufficently large to produce the empirical effective spin-orbit potential. The degeneracy of spin-orbit doublets is removed by including the isoscalar condensate background nucleon self-energies that arise through changes in the quark condensate and the quark density at finite baryon density.

In Table 5 we display the binding energies per nucleon and charge radii of light and medium-heavy nuclei, calculated in the relativistic point-coupling model constrained by in-medium QCD sum rules and chiral perturbation theory, in comparison with experimental values. In addition to the four parameters determined by the nuclear matter equation of state, we have adjusted the isoscalarscalar derivative term in the calculation of ${ }^{16} \mathrm{O}$ and ${ }^{40} \mathrm{Ca}: D_{S}=-0.713 \mathrm{fm}^{4}$. This value of $D_{S}$ is very close to the ones used in the effective interactions of the standard relativistic point-coupling model of Ref. [10]. It is also consistent with the "natural" order of magnitude expected from $D_{S} \sim G_{S} / \Lambda^{2}$. The resulting agreement between the calculated and empirical binding energies and charge radii is indeed very good.

The effect of including the isoscalar condensate background nucleon self-energies is illustrated in Figs. 8 and 9, where we display the neutron and proton singleparticle energies in ${ }^{16} \mathrm{O}$ and ${ }^{40} \mathrm{Ca}$, respectively, in comparison with the corresponding experimental levels. For both nuclei the calculated levels are in excellent agreement with the empirical single-nucleon levels in the vicinity of the Fermi surface. In particular, with the inclusion of the isoscalar condensate self-energies, the model reproduces the empirical energy differences between 
spin-orbit partner states. This is a very important result and it shows that, while nuclear binding and saturation are partially generated by chiral (twopion exchange) fluctuations in our approach, strong scalar and vector fields of equal magnitude and opposite sign, induced by changes of the QCD vacuum in the presence of baryonic matter, generate the large effective spin-orbit potential in finite nuclei. Not surprisingly, the ${ }^{40} \mathrm{Ca}$ spectrum is reminiscent of an underlying pseudo-spin symmetry [46].

The charge density distributions and charge form factors of ${ }^{16} \mathrm{O}$ and ${ }^{40} \mathrm{Ca}$ are displayed in Figs. 10 and 11, respectively. The results obtained in the relativistic point-coupling model, constrained by in-medium QCD sum rules and chiral perturbation theory, are compared with the experimental charge density profiles and form factors [47]. The theoretical charge density distributions are obtained by folding the point proton density distribution with the electric nucleon form factor of dipole form. The corresponding form factors are plotted as functions of the momentum $\boldsymbol{q}[4]$

$$
F_{c h}(\boldsymbol{q})=\frac{1}{Z} \rho_{c h}(\boldsymbol{q})\left[1+\frac{\boldsymbol{q}^{2}}{8\left\langle P_{c m}^{2}\right\rangle}+\ldots\right],
$$

where $\rho_{c h}(\boldsymbol{q})$ is the Fourier transform of the charge density and the second term is a center-of-mass correction. Higher-order effects in $\boldsymbol{q}^{2} / P_{c m}^{2}$ are negligible. We notice the excellent agreement between the calculated and experimental charge form factors for momenta $|\boldsymbol{q}| \leq 2 \mathrm{fm}^{-1}$. When the calculated charge densities are compared to results obtained with standard relativistic mean-field effective interactions, the present results display less pronounced shell effects, in better agreement with empirical data.

In Fig. 12 we compare the single-nucleon spectra of ${ }^{56} \mathrm{Ni}$, calculated in our approach, with the results of a relativistic mean-field calculation using NL3 [48], probably the best non-linear meson-exchange effective interaction. The spectra are in remarkable agreement. All these results demonstrate that in the present approach, based on QCD sum rules and in-medium chiral perturbation theory, and with a small number of model parameters determined directly by these constraints, it is possible to describe symmetric and asymmetric nuclear matter, as well as properties of finite nuclei, at a quantitative level comparable with phenomenological relativistic mean-field models.

\section{$5 \quad$ Summary and conclusions}

The goals of nuclear structure theory have evolved, especially in the last decade, from the macroscopic and microscopic descriptions of structure phe- 
nomena in stable nuclei, towards more exotic nuclei far from the valley of $\beta$ stability, and towards the investigation of the bridge between low-energy QCD and the dynamics of nuclei. New experimental information on exotic nuclei with extreme isospins present serious challenges for traditional nuclear structure models. It is likely that radically improved models, or even completely new theoretical strategies and approaches, will be required as one proceeds to these unexplored region with radioactive nuclear beams.

Models based on effective field theories and density functional theory provide a systematic theoretical framework for studies of nuclear dynamics. By using the important active degrees of freedom at low energies, and by expanding in small parameters determined by the relevant scales, effective Lagrangians are constructed that provide a microscopically consistent, and yet simple and economical approach to the nuclear many-body problem.

In this work we have derived, following Ref. [17], a relativistic nuclear pointcoupling model with density-dependent contact interactions, which emphasizes the connection between nuclear dynamics and two key features of low-energy, non-perturbative QCD: the presence of a non-trivial vacuum characterized by strong condensates and the important role of pionic fluctuations governing the low-energy, long wavelength dynamics according to the rules imposed by spontaneously broken chiral symmetry.

Investigations based on QCD sum rules have shown how large isoscalar scalar and vector nucleon self-energies, that characterize the nuclear ground state, arise in finite-density QCD through changes in the quark condensate and the quark density at finite baryon density. Chiral (pionic) fluctuations superimposed on the condensate background fields play a prominent role for nuclear binding and saturation. These pionic fluctuations are calculated according to the rules of in-medium chiral perturbation theory.

Based on these observations, we have formulated a density-dependent pointcoupling model for nuclear matter and finite nuclei. The coupling parameters are functions of the nucleon density operator $\hat{\rho}$. Their functional dependence is determined from finite density QCD sum rules and in-medium chiral perturbation theory. The strength parameters of the second order isoscalar interaction terms in the Lagrangian include contributions from condensate background fields and pionic (chiral) fluctuations. For the isovector channel it is (so far) assumed that only the pionic fluctuations contribute to the nucleon self-energies.

The resulting nuclear point-coupling model, formally used in the mean-field approximation, but encoding effects beyond mean-field through the density dependent couplings, has been employed in an analysis of the equations of state for symmetric and asymmetric nuclear matter, and of bulk and single- 
nucleon properties of light and medium $N \approx Z$ nuclei. In comparison with purely phenomenological relativistic and non-relativistic mean-field models of nuclear structure, the built-in QCD constraints and the explicit treatment of one- and two-pion exchange significantly reduce the freedom in adjusting parameters and functional forms of density-dependent vertex functions.

A very good equation of state of symmetric nuclear matter is obtained already by equating the isoscalar-scalar, the isoscalar-vector, the isovector-scalar, and the isovector-vector nucleon self-energies in the single-nucleon Dirac equation with those calculated using in-medium chiral perturbation theory up to three loop order in the energy density. The contributions to the energy density originate exclusively from one- and two-pion exchange between nucleons, and they are ordered in powers of the Fermi momentum $k_{f}$ (modulo functions of $\left.k_{f} / m_{\pi}\right)$. The empirical saturation point, the nuclear matter incompressibility, and the asymmetry energy at saturation can be well reproduced at order $\mathcal{O}\left(k_{f}^{5}\right)$ in the chiral expansion with just one single momentum cut-off scale $\Lambda \simeq 0.65$ $\mathrm{GeV}$ which parameterizes unresolved short-distance dynamics.

In the next step we have included the contributions of the condensate background self-energies in the isoscalar-scalar and isoscalar-vector channels. We have considered corrections possibly related to condensates of higher dimension as well as 3-body forces, i.e. terms of order $\rho^{2}$ in the self-energies. The isoscalar condensate background self-energies turn out to be important also for neutron matter. However, details of the isovector interactions (beyond chiral one- and two-pion exchange) must still be improved.

The relativistic nuclear point-coupling model, constrained by in-medium QCD sum rules and chiral perturbation theory, has also been tested in self-consistent calculations of ground state properties of finite nuclei. In the present work we have only considered spherical even-even light and medium-heavy $N \approx Z$ nuclei. Four parameters determine the nuclear matter EOS: the cut-off parameter $\Lambda$ of the in-medium CHPT calculation of two-pion exchange diagrams, the two parameters of the isoscalar condensate background nucleon self-energies linear in the corresponding densities: $G_{S}^{(0)}$ and $G_{V}^{(0)}$, and the strength parameter $g_{V}^{(1)}$ of the quadratic term of the isoscalar vector self-energy. For finite nuclei we adjust in addition the strength parameter of the isoscalar-scalar derivative interaction term in the Lagrangian. This brings the total number of adjustable parameters to five. It has been emphasized that the empirical data set of $N \approx Z$ finite nuclei can only constrain five or six parameters in the general expansion of an effective Lagrangian in powers of the fields and their derivatives. In particular, only one parameter of the derivative terms can be determined by the binding energies and radii of spherical nuclei. The $G_{S, V}^{(0)}$ turn out to be so remarkably close to leading order QCD sum rule estimates that one could have guessed their values right from the beginning. The remaining three parameters (the high momentum scale reflecting unresolved 
short-distance physics, a "three body" contribution to the nucleon self-energy and a surface (derivative) term) behave "naturally" according to the powercounting hierarchy of effective field theory. Of course, questions concerning systematic convergence of the in-medium chiral loop expansion still remain and need to be further explored.

An important result of the present analysis is that chiral (two-pion exchange) fluctuations are consistently included in the nuclear binding and in the saturation mechanism. These fluctuations are superimposed on the condensate background scalar and vector fields of equal magnitude and opposite sign, induced by changes of the QCD vacuum in the presence of baryonic matter. They drive the large spin-orbit splitting in finite nuclei. The calculated neutron and proton single-particle energies in ${ }^{16} \mathrm{O}$ and ${ }^{40} \mathrm{Ca}$ are in excellent agreement with the empirical single-nucleon levels in the vicinity of the Fermi surface, including the empirical energy differences between spin-orbit partner states.

This work has demonstrated that an approach to nuclear dynamics, constrained by the chiral symmetry breaking pattern and the condensate structure of low-energy QCD, can describe symmetric and asymmetric nuclear matter, as well as properties of finite nuclei, at a quantitative level comparable with phenomenological relativistic and non-relativistic mean-field models. This is a promising result, which opens perspectives of bridging the gap between basic features of low-energy, non perturbative QCD and the rich nuclear phenomenology.

\section{References}

[1] B.D. Serot and J.D. Walecka, Adv. Nucl. Phys. 16, 1 (1986); Int. J. Mod. Phys. E6, 515 (1997).

[2] P. Ring, Progr. Part. Nucl. Phys. 37, 193 (1996).

[3] R.J. Furnstahl and B.D. Serot, Nucl. Phys. A673, 298 (2000); Comments Nucl. Part. Phys. 2, A23 (2000).

[4] R.J. Furnstahl, B.D. Serot, and H.-B. Tang, Nucl. Phys. A615, 441 (1997); A640, 505 (E) (1998).

[5] W. Lin and B.D. Serot, Phys. Lett. B233, 23 (1989); Nucl. Phys. A512, 637 (1990).

[6] R.J. Furnstahl and B.D. Serot, Nucl. Phys. A671, 447 (2000).

[7] A. Manohar and H. Georgi, Nucl. Phys. B234, 189 (1984).

[8] J.L. Friar, D.G. Madland and B.W. Lynn, Phys. Rev. C53, 3085 (1996). 
[9] J. J. Rusnak and R. J. Furnstahl, Nucl. Phys. A627, 495 (1997).

[10] T. Burvenich, D. G. Madland, J. A. Maruhn and P. G. Reinhard, Phys. Rev. C65, 044308 (2001).

[11] T.D. Cohen, R.J. Furnstahl and D.K. Griegel, Phys. Rev. Lett. 67, 961 (1991); Phys. Rev. C45, 1881 (1992).

[12] E.G. Drukarev and E.M. Levin, Nucl. Phys. A511, 679 (1990); Prog. Part. Nucl. Phys. 27, 77 (1991).

[13] X. Jin, M. Nielsen, T.D. Cohen, R.J. Furnstahl and D.K. Griegel, Phys. Rev. C49, 464 (1994).

[14] H. W. Hammer and R. J. Furnstahl, Nucl. Phys. A678, 277 (2000).

[15] N. Kaiser, S. Fritsch, and W. Weise, Nucl. Phys. A697, 255 (2002).

[16] N. Kaiser, S. Fritsch and W. Weise, Nucl. Phys. A700, 343 (2002).

[17] P. Finelli, N. Kaiser, D. Vretenar and W. Weise, Eur. Phys. J. A17, 573 (2003).

[18] R.J. Furnstahl, H.-B. Tang, and B.D. Serot, Phys. Rev. C52, 1368 (1995).

[19] C. Fuchs, H. Lenske, and H.H. Wolter, Phys. Rev. C52, 3043 (1995).

[20] P. Manakos and T. Mannel, Z. Phys. A334, 481 (1989).

[21] D.G. Madland, B.A. Nikolaus and T. Hoch, Phys. Rev. C46, 1757 (1992).

[22] F. de Jong and H. Lenske, Phys. Rev. C57, 3099 (1998).

[23] F. Hofmann, C.M. Keil and H. Lenske, Phys. Rev. C64, 034314 (2001).

[24] S. Typel and H.H. Wolter, Nucl. Phys. A656, 331 (1999).

[25] T. Niksic, D. Vretenar, P. Finelli and P. Ring, Phys. Rev. C66, 024306 (2002).

[26] A. Pich and J. Prades, Nucl. Phys. Proc. Suppl. 86, 236 (2000); B. L. Ioffe, Phys. At. Nucl. (Yad. Fiz.) 66, 30 (2003), and refs. therein.

[27] B. L. Ioffe, Nucl. Phys. B188, 317 (1981).

[28] J. Gasser, H. Leutwyler and M. Sarinio, Phys. Lett. B253, 252 (1991).

[29] B. Friedman and V.R. Pandharipande, Nucl. Phys. A361, 502 (1981).

[30] D. Vretenar, G.A. Lalazissis, R. Behnsch, W. Pöschl and P. Ring, Nucl. Phys. A621, 853 (1997).

[31] Zhong-yu Ma, Nguyen Van Giai, A. Wandelt, D. Vretenar and P. Ring, Nucl. Phys. A686, 173 (2001).

[32] C.-H. Lee, T.T.S. Kuo, G.Q. Li and G.E. Brown, Phys. Rev. C57, 3488 (1998).

[33] R.J. Furnstahl, Nucl. Phys. A706, 85 (2002). 
[34] V.E. Starodubsky and N.M. Hintz, Phys. Rev. C49, 2118 (1994).

[35] A. Krasznahorkay et al., Nucl. Phys. A567, 521 (1994).

[36] W. Zuo, I. Bombaci and U. Lombardo, Phys. Rev. C60, 024605 (1999).

[37] J. V. Steele, arXiv:nucl-th/0010066.

[38] T. Gross-Boelting, C. Fuchs and A. Faessler, Nucl. Phys. A648, 105 (1999).

[39] S. K. Bogner, T. T. Kuo and A. Schwenk, Phys. Rept. 386, 1 (2003); A. Schwenk, G. E. Brown and B. Friman, Nucl. Phys. A703, 745 (2002).

[40] W. Kohn and L. J. Sham, Phys. Rev. A140, 1133 (1965).

[41] W. Kohn, Rev. Mod. Phys. 71, 1253 (1999).

[42] R. M. Dreizler and E. K. U. Gross, Density Functional Theory, (Springer, 1990).

[43] Y.K. Gambhir, P. Ring and A. Thimet, Ann. Phys. (N.Y.) 198, 132 (1990).

[44] B.D. Serot and J.D. Walecka, Phys. Lett. B87, 172 (1979).

[45] R. Brockmann and W. Weise, Phys. Rev. C16, 677 (1977).

[46] J. N. Ginocchio, Phys. Rev. C66, 064312 (2002).

[47] H. de Vries, C.W. de Jager, and C. de Vries, At. Data Nucl. Data Tables 36, 495 (1987).

[48] G.A. Lalazissis, J. König, and P. Ring, Phys. Rev. C55, 540 (1997). 
Table 1

The coefficients of the expansion (40) up to order $k_{f}^{5}$ of the in-medium CHPT isoscalar-vector, isoscalar-scalar, isovector-vector, and isovector-scalar nucleon selfenergies.

\begin{tabular}{|c|c|c|c|c|}
\hline & V-VERTEX & S-VERTEX & TV-VERTEX & TS-VERTEX \\
\hline$c_{30}$ & 3.96669 & 3.97078 & -4.00813 & -4.12669 \\
$c_{31}$ & -7.55255 & -7.55255 & 6.54555 & 6.54555 \\
$c_{32}$ & 0.762611 & 0.60693 & -0.544615 & -1.73796 \\
$c_{3 L}$ & 0.29288 & 0.267627 & -0.370303 & -0.563877 \\
$c_{40}$ & 8.42137 & 8.11713 & -2.4575 & -2.97905 \\
$c_{50}$ & 10.2384 & 12.0729 & -26.8119 & -23.591 \\
$c_{5 L}$ & 1.16164 & 1.92167 & -7.81327 & -6.50123 \\
\hline
\end{tabular}

Table 2

The coefficients of the expansion of the in-medium CHPT self energies (41) - (44) in powers of the baryon density, for the value of the cut-off parameter $\Lambda=646.3$ $\mathrm{MeV}$. In this case we assumed $N=Z$, neglecting isovector contributions.

\begin{tabular}{|c|c|c|c|}
\hline$c_{s 1}$ & $-2.805 \mathrm{fm}^{2}$ & $c_{t s 1}$ & $1.491 \mathrm{fm}^{2}$ \\
$c_{s 2}$ & $2.738 \mathrm{fm}^{3}$ & $c_{t s 2}$ & $-1.005 \mathrm{fm}^{3}$ \\
$c_{s 3}$ & $1.346 \mathrm{fm}^{4}$ & $c_{t s 3}$ & $-1.550 \mathrm{fm}^{4}$ \\
$c_{v 1}$ & $-2.718 \mathrm{fm}^{2}$ & $c_{t v 1}$ & $2.249 \mathrm{fm}^{2}$ \\
$c_{v 2}$ & $2.841 \mathrm{fm}^{3}$ & $c_{t v 2}$ & $-0.829 \mathrm{fm}^{3}$ \\
$c_{v 3}$ & $1.325 \mathrm{fm}^{4}$ & $c_{t v 3}$ & $-1.595 \mathrm{fm}^{4}$ \\
\hline
\end{tabular}


Table 3

Nuclear matter saturation properties: binding energy per nucleon, saturation density and incompressibility. The first row corresponds to the in-medium CHPT calculation including one- and two-pion exchange between nucleons [15]. The EOS displayed in the second row (PC-dd) is obtained when the resulting CHPT nucleon self-energies are mapped on the self-energies of the relativistic point-coupling model with density dependent couplings.

\begin{tabular}{|c|c|c|c|}
\hline MODEL & E/A $(\mathrm{MeV})$ & $\rho_{\text {sat }}\left(\mathrm{fm}^{-3}\right)$ & $K_{0}(\mathrm{MeV})$ \\
\hline CHPT [15] & -15.26 & 0.178 & 255 \\
PC-dd & -14.51 & 0.175 & 235 \\
\hline
\end{tabular}

Table 4

Nuclear matter saturation properties calculated in the relativistic point-coupling model constrained by in-medium QCD sum rules and chiral perturbation theory. In addition to the chiral one- and two-pion exchange contribution to the density dependence of the coupling parameters, the nuclear matter EOS shown in the first row includes also the isoscalar condensate background nucleon self-energies linear in the corresponding densities. The EOS displayed in the second row is calculated by including also the non-linear " 3 -body" contribution (proportional to $\rho^{2}$ ) in the isoscalar vector condensate self-energy.

\begin{tabular}{|c|c|c|c|c|}
\hline & $\mathrm{E} / \mathrm{A}(\mathrm{MeV})$ & $\rho_{\text {sat }}\left(\mathrm{fm}^{-3}\right)$ & $K_{0}(\mathrm{MeV})$ & $M^{*} / M$ \\
\hline LINEAR & -15.97 & 0.148 & 283 & 0.753 \\
NON-LINEAR & -15.76 & 0.151 & 332 & 0.620 \\
\hline
\end{tabular}


Table 5

Binding energies per nucleon and charge radii of light and medium-heavy nuclei, calculated in the relativistic point-coupling model constrained by in-medium QCD sum rules and chiral perturbation theory, are compared with experimental values.

\begin{tabular}{|r|c|c|c|c|}
\hline & $E / A^{\exp }(\mathrm{MeV})$ & $E / A(\mathrm{MeV})$ & $r_{c}^{\exp }\left(\mathrm{fm}^{-3}\right)$ & $r_{c}\left(\mathrm{fm}^{-3}\right)$ \\
\hline${ }^{16} \mathrm{O}$ & 7.976 & 8.019 & 2.730 & 2.724 \\
${ }^{40} \mathrm{Ca}$ & 8.551 & 8.494 & 3.485 & 3.447 \\
${ }^{42} \mathrm{Ca}$ & 8.617 & 8.482 & 3.513 & 3.450 \\
${ }^{48} \mathrm{Ca}$ & 8.666 & 8.396 & 3.484 & 3.476 \\
${ }^{42} \mathrm{Ti}$ & 8.260 & 8.127 & - & 3.536 \\
${ }^{50} \mathrm{Ti}$ & 8.756 & 8.468 & - & 3.549 \\
${ }^{52} \mathrm{Cr}$ & 8.776 & 8.494 & 3.647 & 3.614 \\
${ }^{58} \mathrm{Ni}$ & 8.732 & 8.445 & 3.783 & 3.748 \\
${ }^{64} \mathrm{Ni}$ & 8.777 & 8.451 & 3.868 & 3.836 \\
${ }^{88} \mathrm{Sr}$ & 8.733 & 8.439 & 4.206 & 4.205 \\
${ }^{90} \mathrm{Zr}$ & 8.710 & 8.443 & 4.272 & 4.252 \\
\hline
\end{tabular}




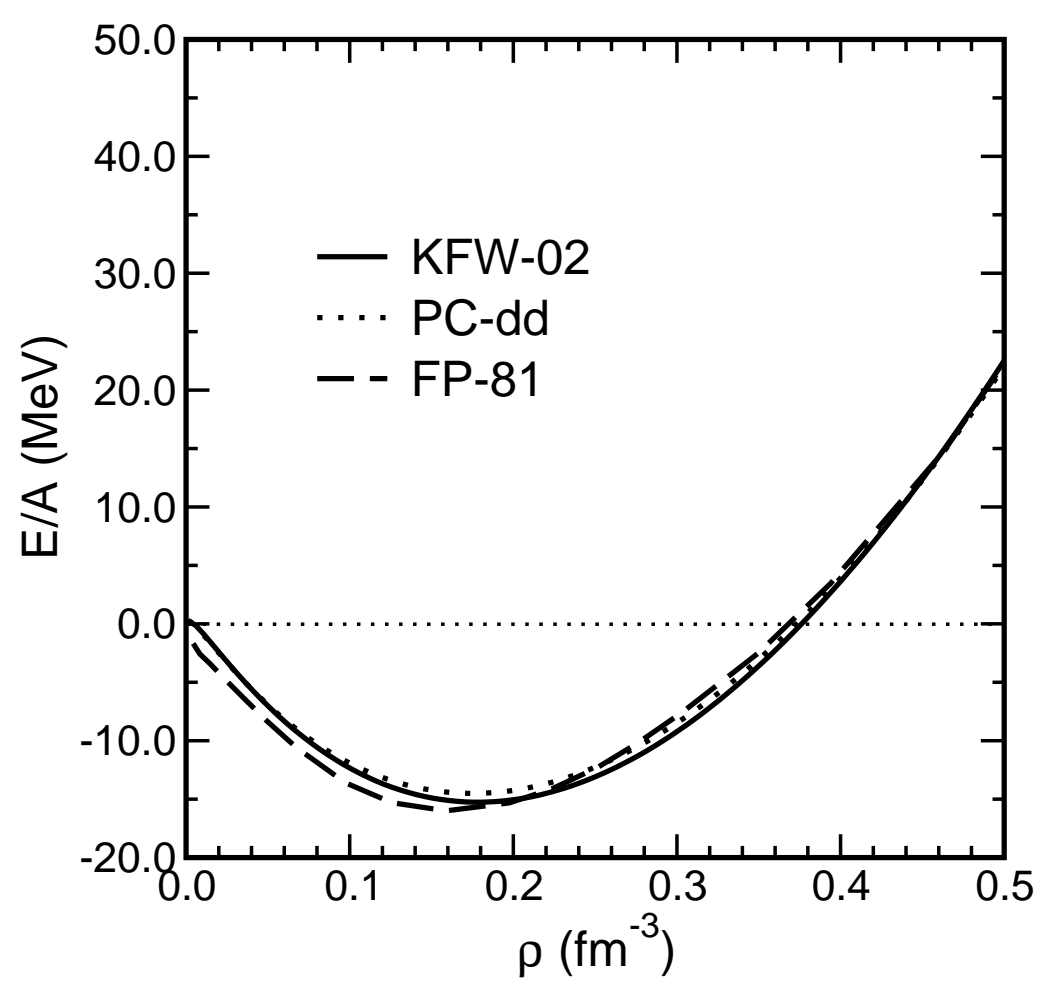

Fig. 1. Binding energy per nucleon for symmetric nuclear matter as a function of baryon density. The solid curve (KFW-02) is the EOS calculated in Ref. [15] by using in-medium CHPT for one- and two-pion exchange between nucleons. The EOS displayed by the dotted curve (PC-dd) is obtained when the resulting CHPT nucleon self-energies are mapped on the self-energies of the relativistic point-coupling model with density dependent couplings. FP-81 denotes the microscopic many-body nuclear matter equation of state of Friedman and Pandharipande [29], shown for comparison. 


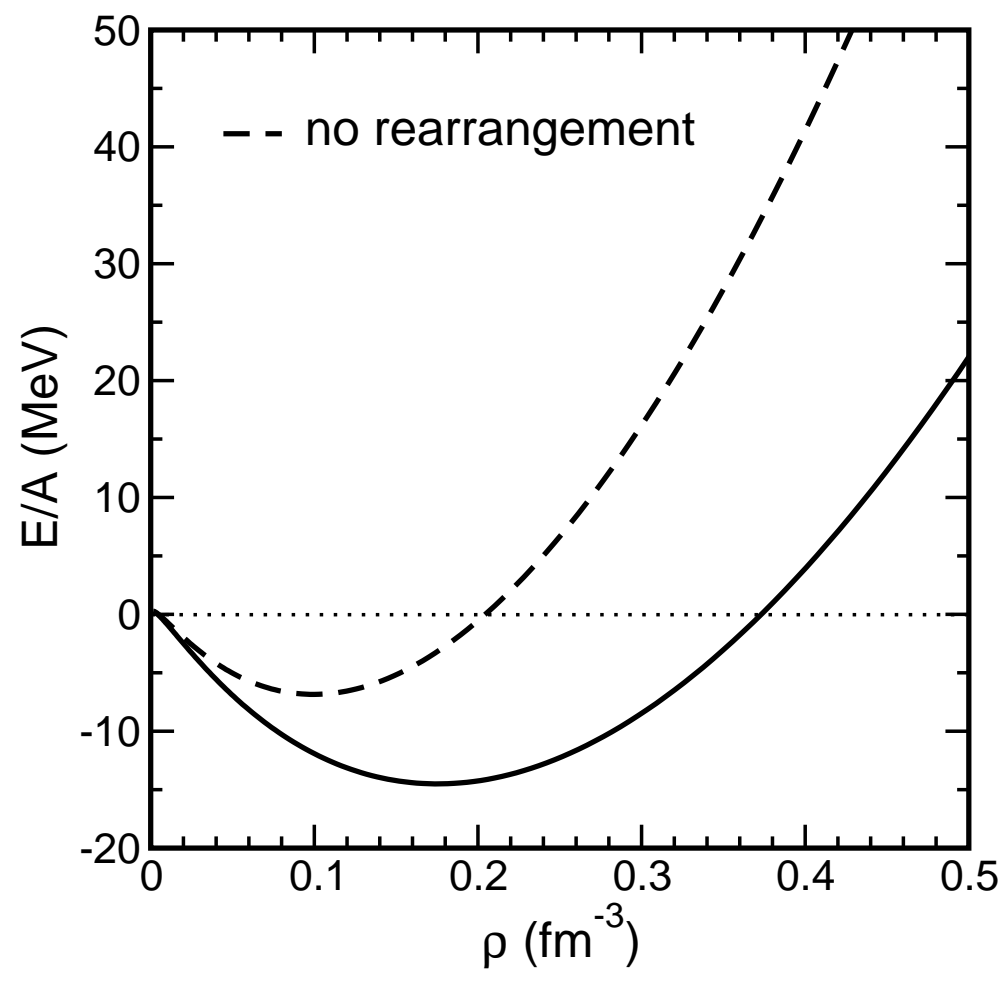

Fig. 2. Equation of state of symmetric nuclear matter calculated in the relativistic point-coupling model with and without the rearrangement contribution to the isoscalar vector self-energy. 


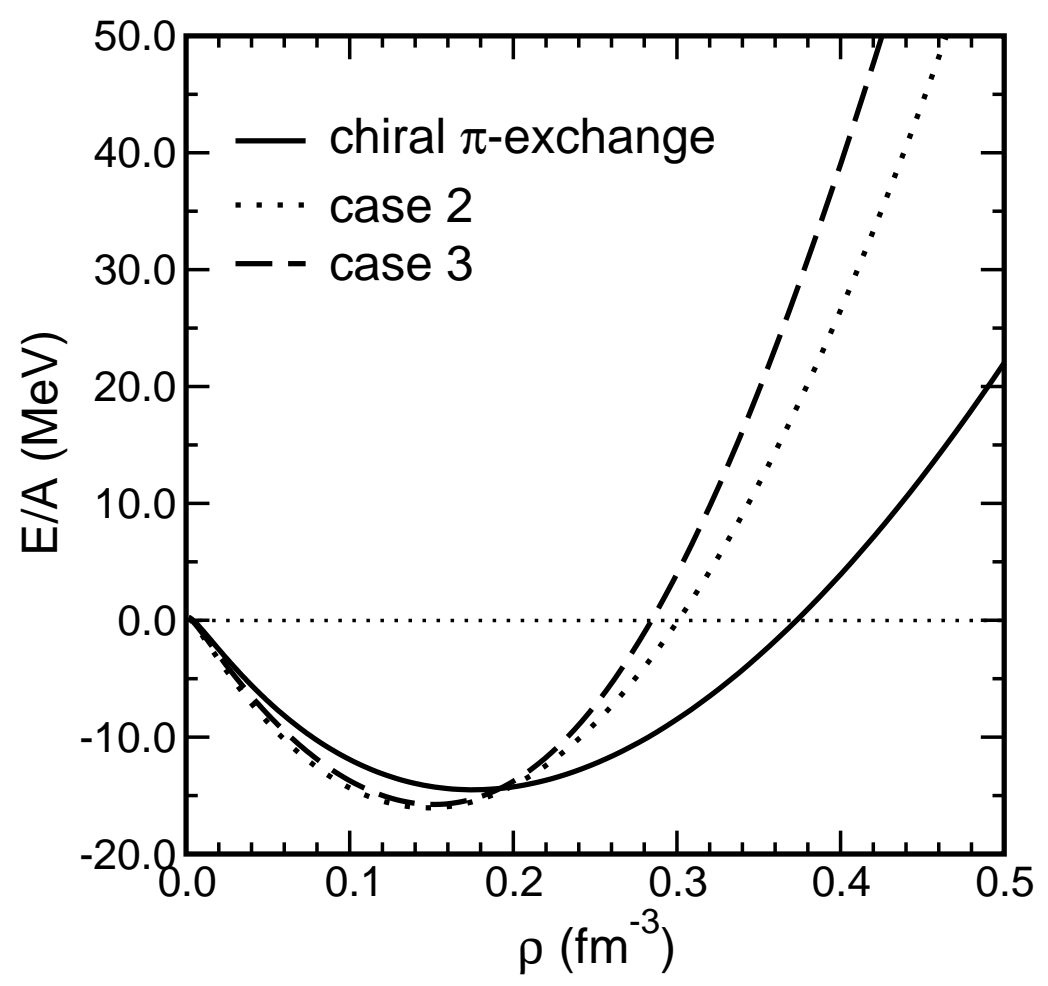

Fig. 3. Binding energy per nucleon for symmetric nuclear matter as a function of the baryon density, calculated from chiral one- and two-pion exchange between nucleons (case 1, solid curve), by adding the isoscalar condensate background nucleon self-energies linear in the corresponding densities, with $G_{V}^{(0)}=-G_{S}^{(0)}=7 \mathrm{fm}^{2}$ (case 2 , dotted curve), and finally by including also the non-linear contribution $g_{V}^{(1)} \rho^{2}$ to the isoscalar vector condensate self-energy, with $G_{V}^{(0)}=11 \mathrm{fm}^{2}, G_{S}^{(0)}=-12 \mathrm{fm}^{2}$ (case 3, dashed curve). 


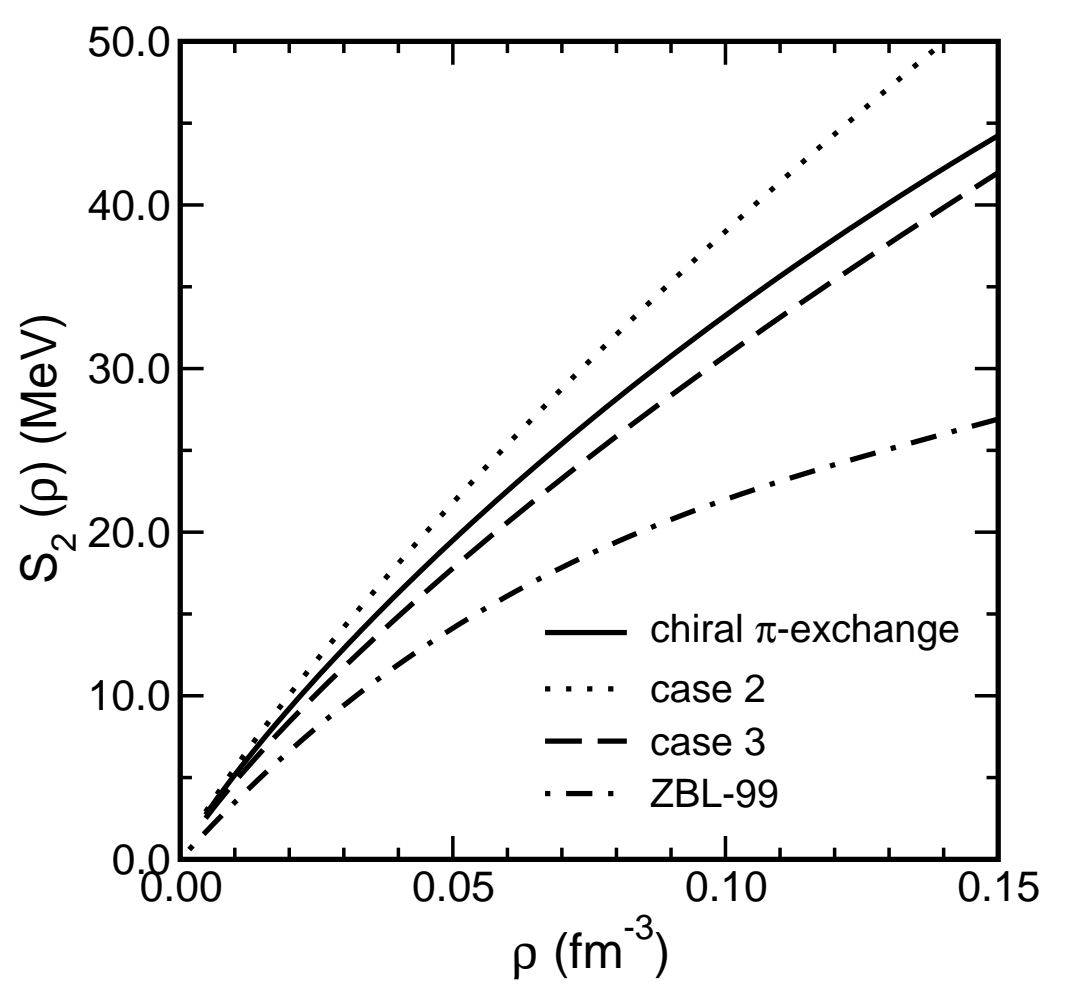

Fig. 4. $S_{2}(\rho)$ coefficient (55) of the quadratic term of the energy per particle of asymmetric nuclear matter. The three curves correspond to calculations with only chiral one- and two-pion exchange between nucleons, with the inclusion of leading order isoscalar condensate background nucleon self-energies, and finally with the contribution of the non-linear vector condensate nucleon self-energy. Cases 2 and 3 are explained in the caption of Fig. 3 and ZBL-99 denotes the asymmetry energy curve obtained in the non-relativistic Brueckner-Hartree-Fock calculation of Ref. [36]. 


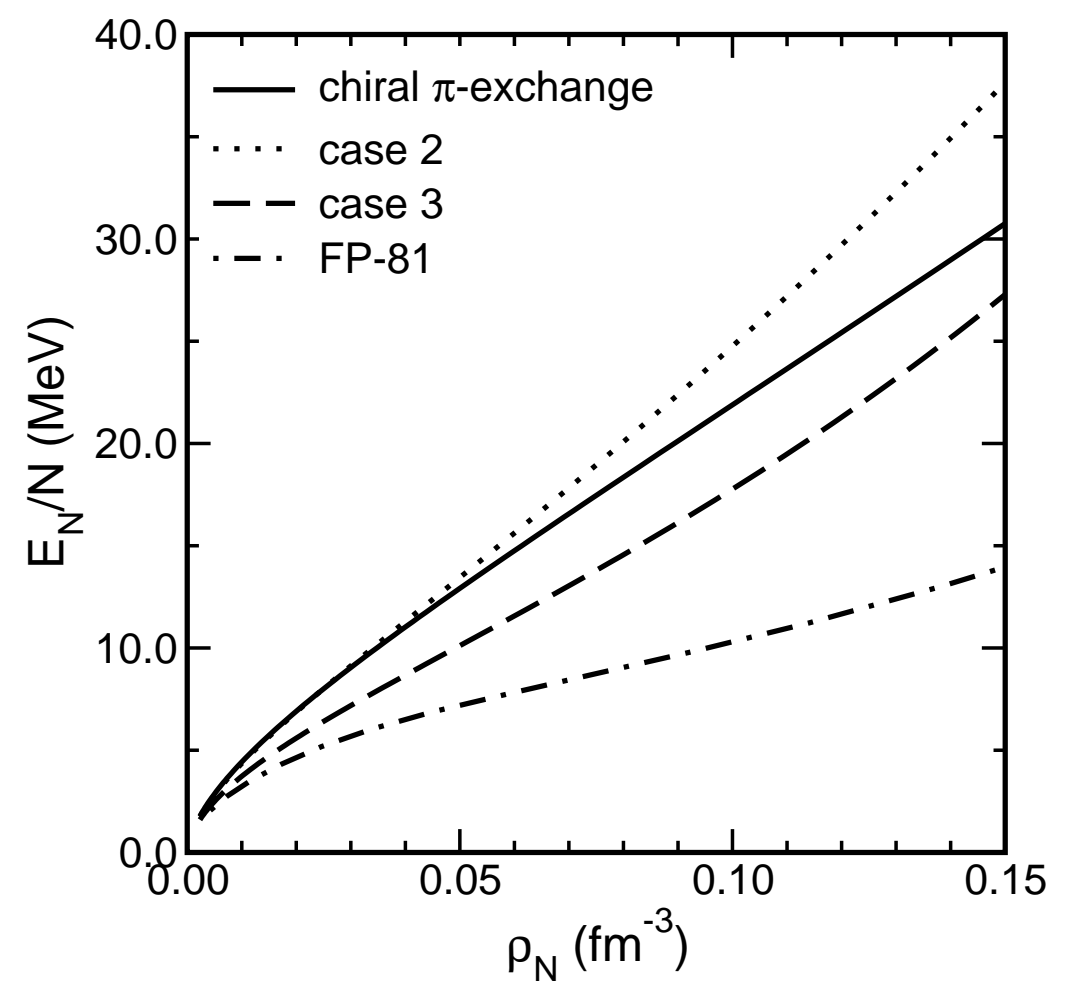

Fig. 5. Energy per particle of neutron matter as a function of the neutron density. The results are obtained from chiral one- and two-pion exchange between nucleons (solid curve), by adding the isoscalar condensate background nucleon self-energies linear in the corresponding densities (case 2, dotted curve), and finally by including also the non-linear contribution to the isoscalar vector condensate self-energy (case 3, dashed curve). Cases 2 and 3 are the same as in the caption of Fig. 3. This is shown in comparison with the microscopic many-body neutron matter equation of state of Friedman and Pandharipande [29]. 


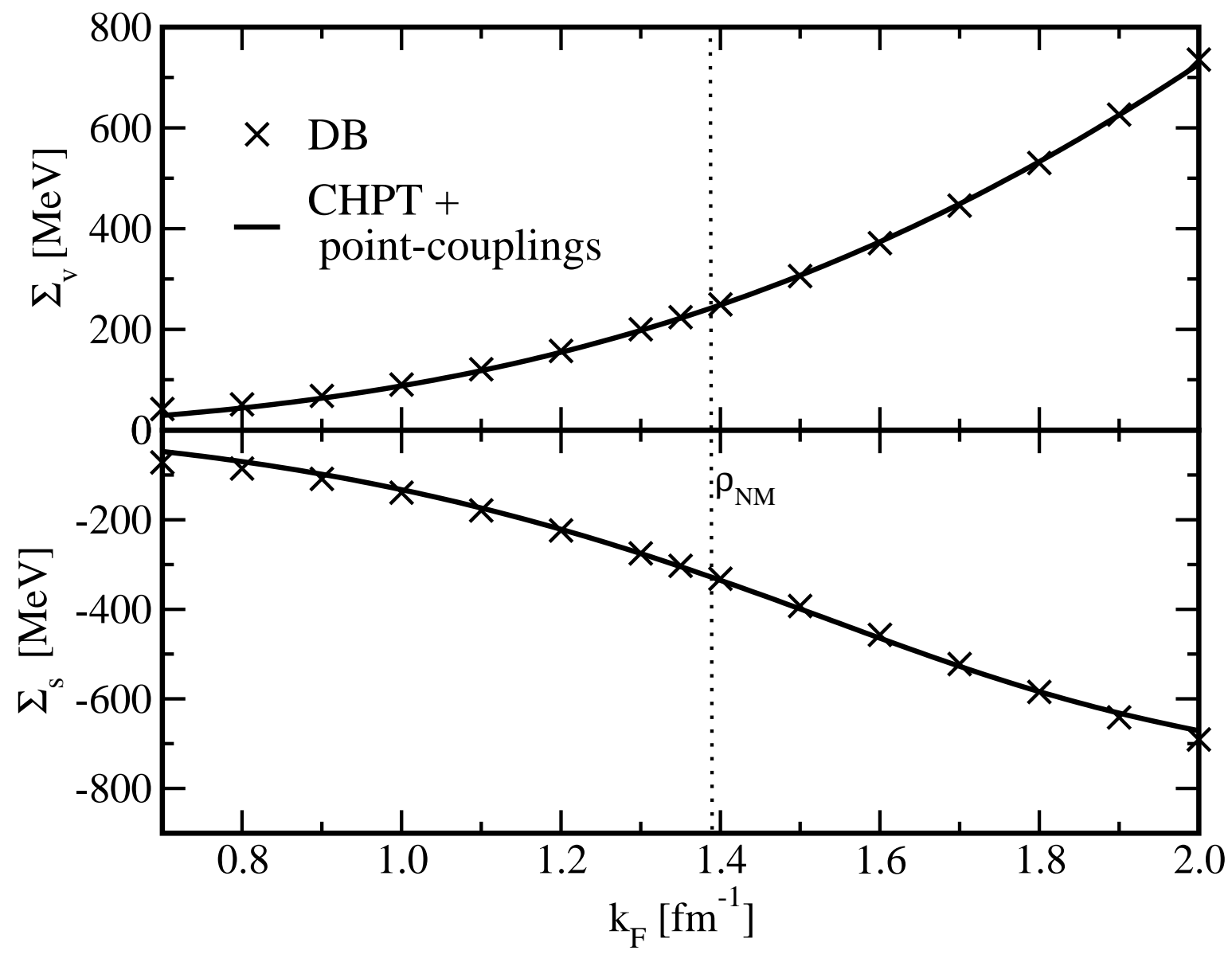

Fig. 6. Comparison of the $k_{f}$-dependence of the isoscalar vector and scalar self-energies resulting from a Dirac-Brueckner G-matrix calculation (solid lines: DB [38]), with the self-energies generated from in-medium chiral perturbation theory (dashed lines: CHPT + point-couplings up to 3-loop order in the energy density). 


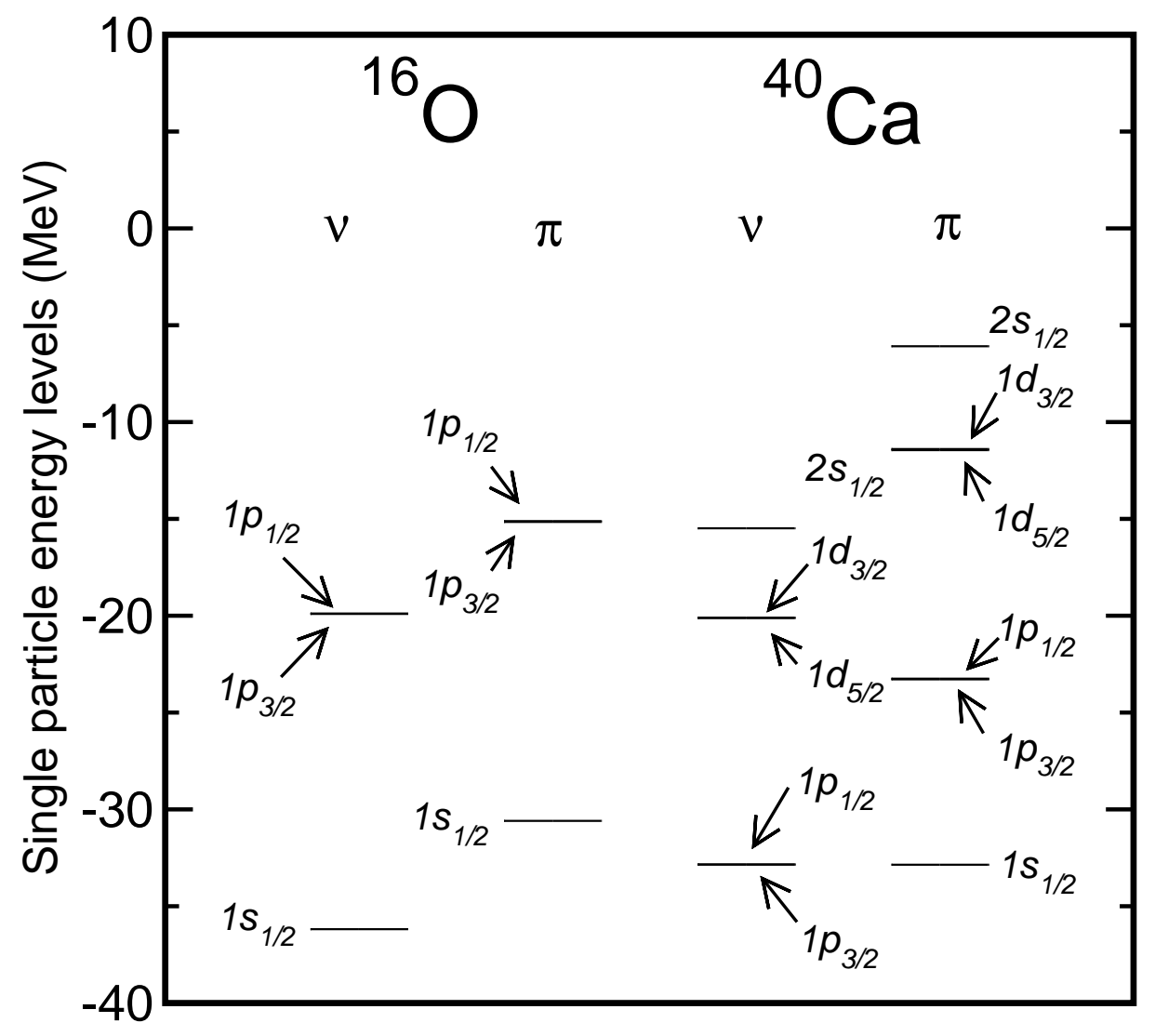

Fig. 7. Neutron and proton single-particle levels in ${ }^{16} \mathrm{O}$ and ${ }^{40} \mathrm{Ca}$ calculated in the relativistic point-coupling model. The density dependent coupling strengths include only the contribution from chiral one- and two-pion exchange between nucleons. 


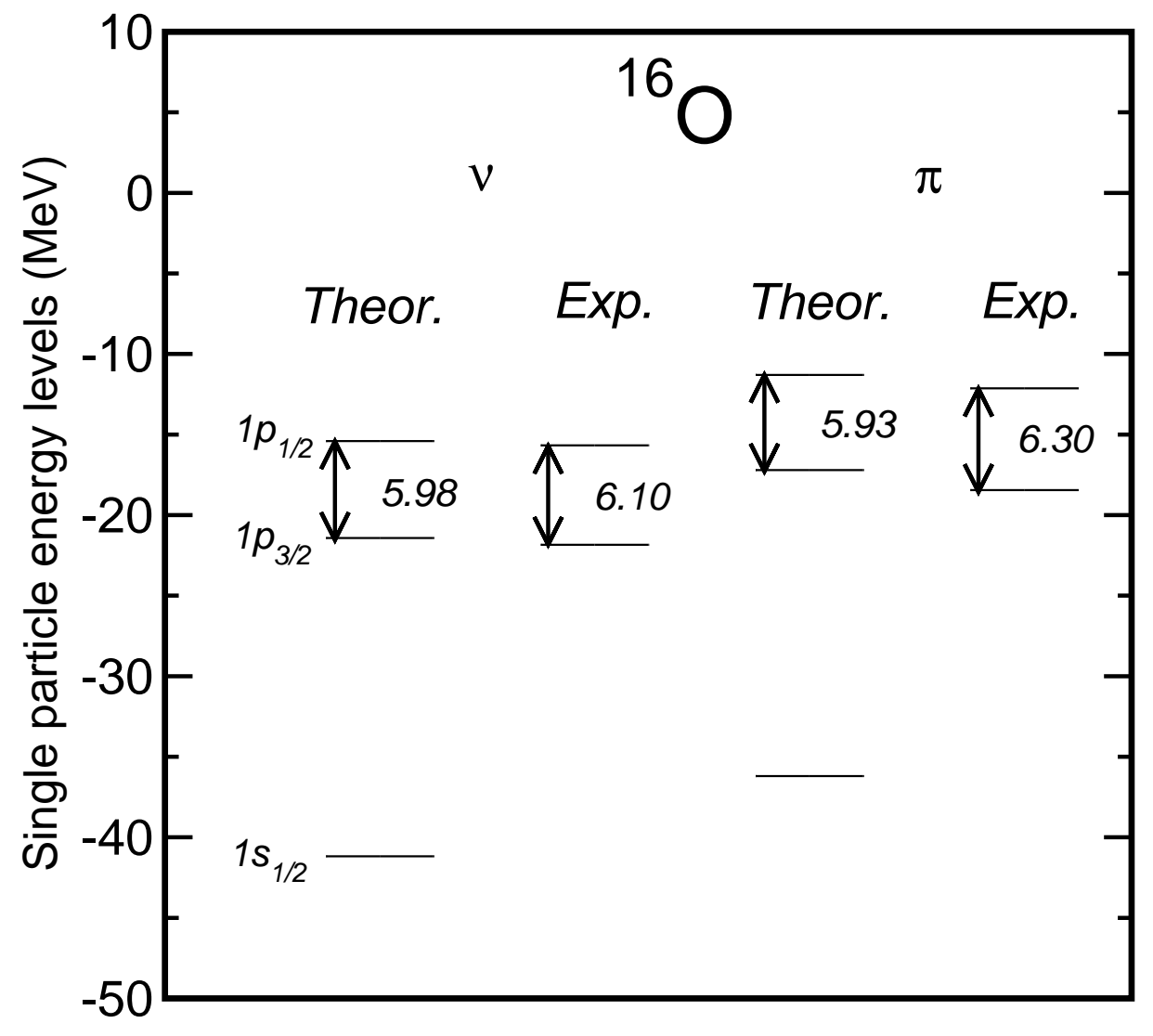

Fig. 8. The neutron and proton single-particle levels in ${ }^{16} \mathrm{O}$ calculated in the relativistic point-coupling model, are shown in comparison with experimental levels. The calculation is performed by including both the contributions of chiral pion-nucleon exchange and of the isoscalar condensate self-energies in the density dependent coupling strengths. 


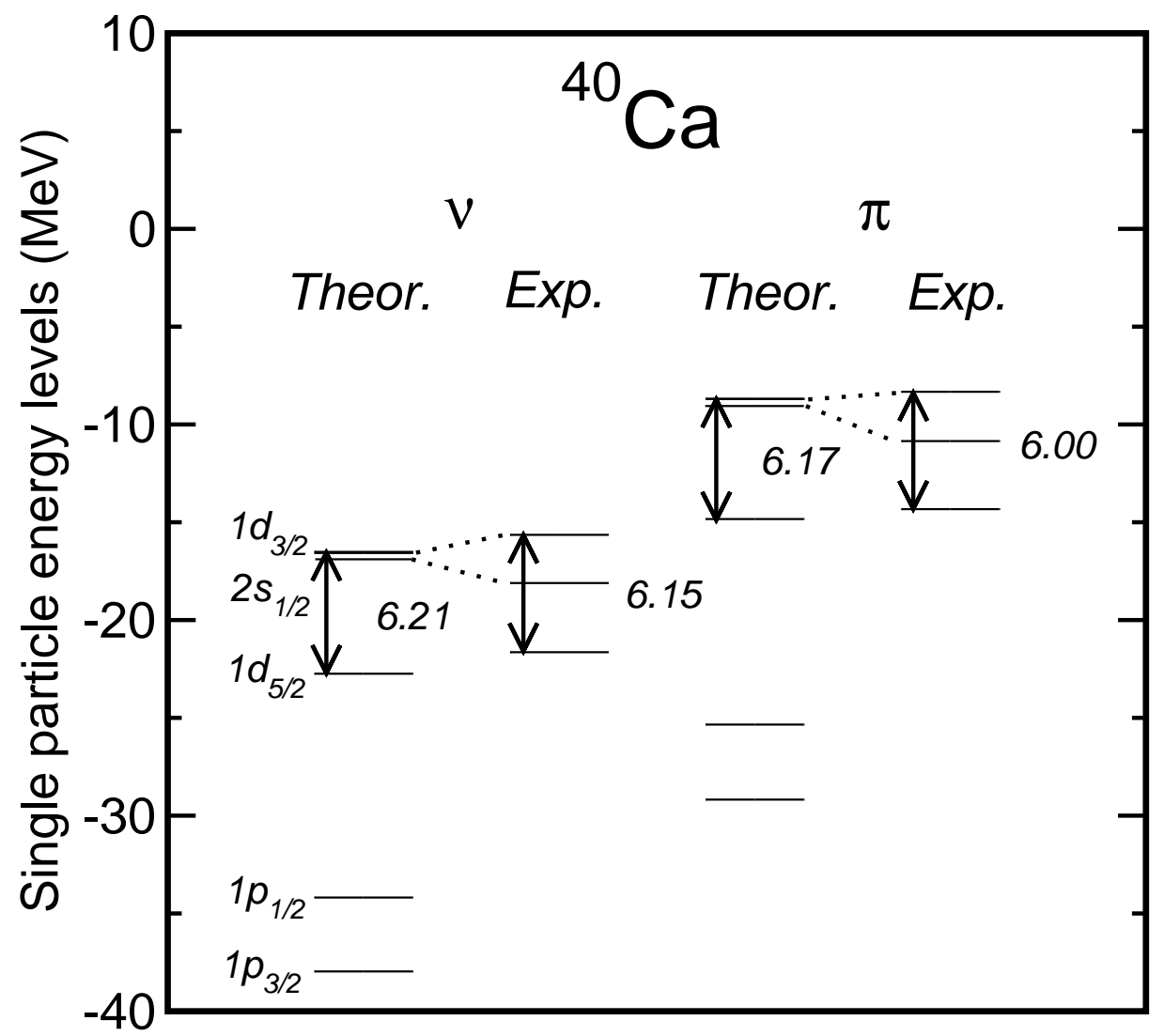

Fig. 9. Same as in Fig. 8, but for ${ }^{40} \mathrm{Ca}$. 

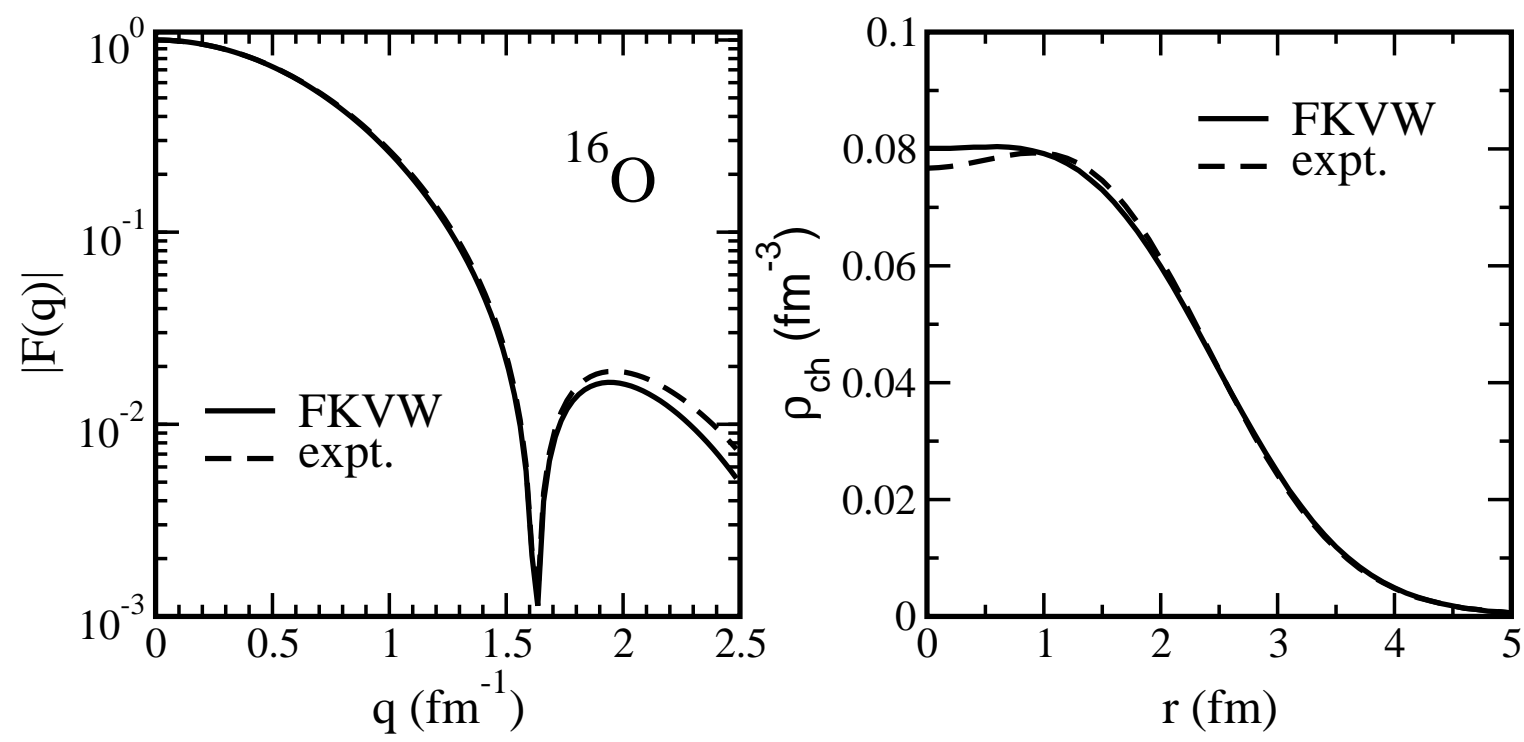

Fig. 10. Charge form factor (left panel) and charge density distribution (right panel) of ${ }^{16} \mathrm{O}$. The results obtained in the relativistic point-coupling model, constrained by in-medium QCD sum rules and chiral perturbation theory, are compared with experimental data [47].
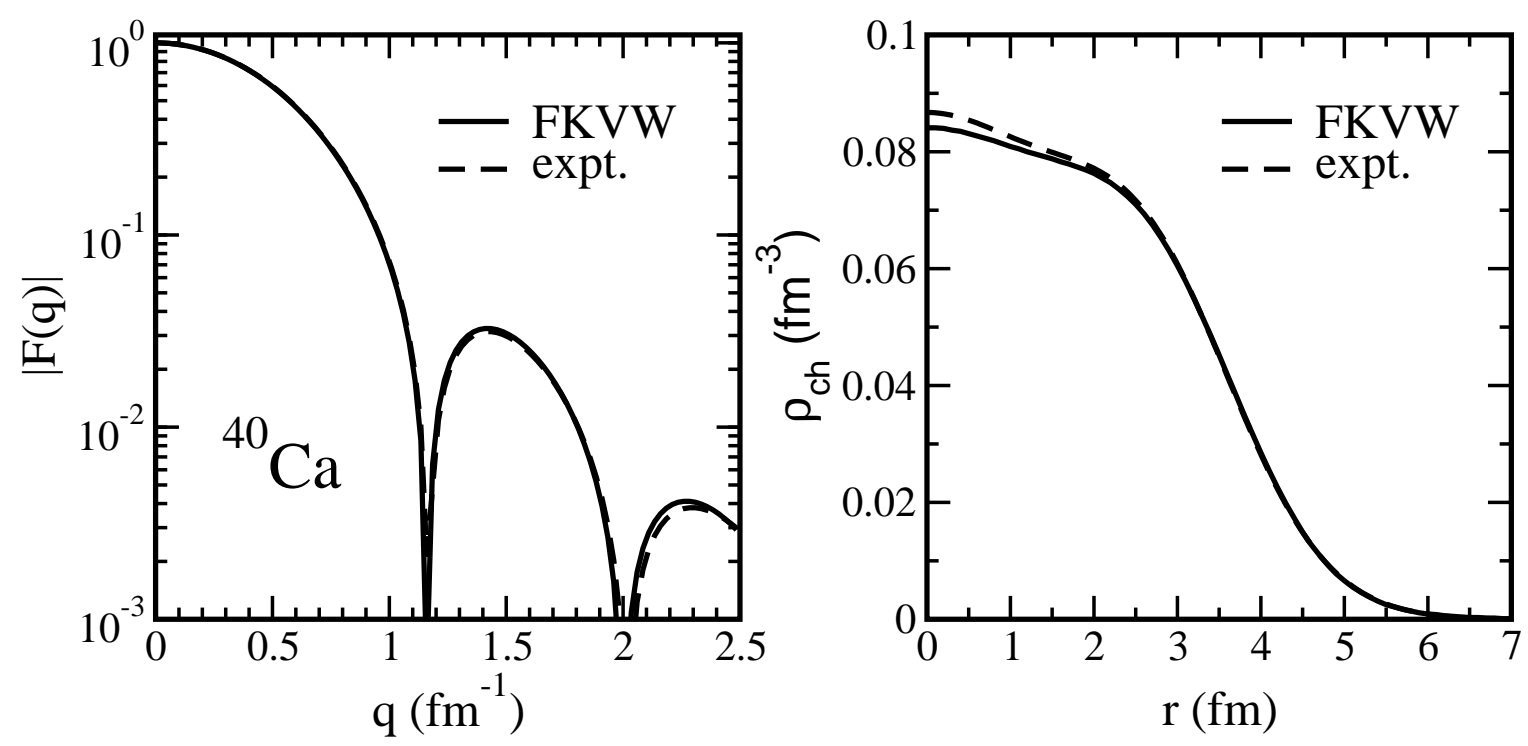

Fig. 11. Same as in Fig. 10, but for ${ }^{40} \mathrm{Ca}$. 


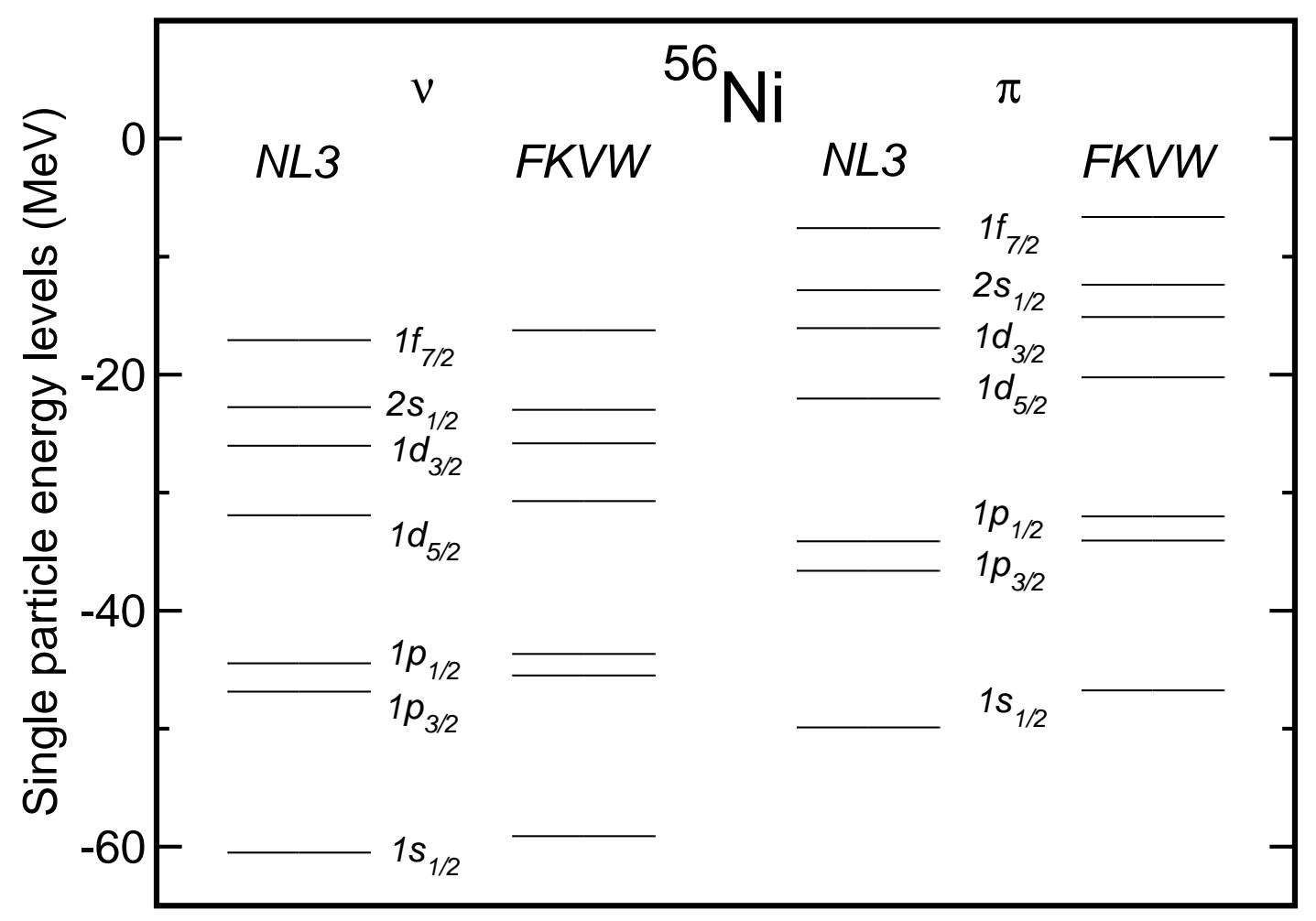

Fig. 12. Neutron and proton single-particle spectra of ${ }^{56} \mathrm{Ni}$, calculated with the standard relativistic mean-field model NL3 effective interaction [48], and with the relativistic point-coupling model constrained by in-medium QCD sum rules and chiral perturbation theory (FKVW). 